\title{
Dynamics of mobile genetic elements of Listeria monocytogenes persisting in ready- to-eat seafood processing plants in France
}

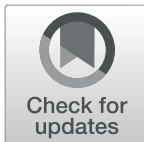

Federica Palma ${ }^{1 *} \mathbb{D}$, Thomas Brauge ${ }^{2}$, Nicolas Radomski ${ }^{1}$, Ludovic Mallet $^{1}$, Arnaud Felten ${ }^{1}$, Michel-Yves Mistou ${ }^{1,3}$, Anne Brisabois ${ }^{1,2}$, Laurent Guillier ${ }^{1}$ and Graziella Midelet-Bourdin ${ }^{2}$

\begin{abstract}
Background: Listeria monocytogenes Clonal Complexes (CCs) have been epidemiologically associated with foods, especially ready-to-eat (RTE) products for which the most likely source of contamination depends on the occurrence of persisting clones in food-processing environments (FPES). As the ability of L. monocytogenes to adapt to environmental stressors met in the food chain challenges the efforts to its eradication from FPEs, the threat of persistent strains to the food industry and public health authorities continues to rise. In this study, 94 food and FPEs L. monocytogenes isolates, representing persistent subtypes contaminating three French seafood facilities over 2-6 years, were whole-genome sequenced to characterize their genetic diversity and determine the biomarkers associated with long-term survival in FPEs.
\end{abstract}

Results: Food and FPEs isolates belonged to five CCs, comprising long-term intra- and inter-plant persisting clones. Mobile genetic elements (MGEs) such as plasmids, prophages and transposons were highly conserved within CCs, some of which harboured genes for resistance to chemical compounds and biocides used in the processing plants. Some of these genes were found in a $90.8 \mathrm{kbp}$ plasmid, predicted to be" mobilizable", identical in isolates from CC204 and CC155, and highly similar to an $81.6 \mathrm{kbp}$ plasmid from isolates belonging to CC7. These similarities suggest horizontal transfer between isolates, accompanied by deletion and homologous recombination in isolates from CC7. Prophage profiles characterized persistent clonal strains and several prophage-loci were plant-associated. Notably, a persistent clone from CC101 harboured a novel $31.5 \mathrm{kbp}$ genomic island that we named Listeria genomic island 3 (LGI3), composed by plant-associated loci and chromosomally integrating cadmium-resistance determinants cadAlC.

Conclusions: Genome-wide analysis indicated that inter- and intra-plant persisting clones harbour conserved MGEs, likely acquired in FPEs and maintained by selective pressures. The presence of closely related plasmids in $L$. monocytogenes CCS supports the hypothesis of horizontal gene transfer conferring enhanced survival to FPEassociated stressors, especially in hard-to-clean harbourage sites. Investigating the MGEs evolutionary and transmission dynamics provides additional resolution to trace-back potentially persistent clones. The biomarkers herein discovered provide new tools for better designing effective strategies for the removal or reduction of resident $L$. monocytogenes in FPEs to prevent contamination of RTE seafood.

Keywords: Listeria monocytogenes, Persistent clones, Food processing plant, Comparative genomics, Mobile genetic elements, Horizontal plasmid transfer, Prophage profiling, Genomic island

\footnotetext{
* Correspondence: federica.palma@anses.fr

${ }^{1}$ ANSES, Laboratory for Food Safety, University Paris-Est, Maisons-Alfort,

France

Full list of author information is available at the end of the article
}

(c) The Author(s). 2020 Open Access This article is distributed under the terms of the Creative Commons Attribution 4.0 International License (http://creativecommons.org/licenses/by/4.0/), which permits unrestricted use, distribution, and reproduction in any medium, provided you give appropriate credit to the original author(s) and the source, provide a link to the Creative Commons license, and indicate if changes were made. The Creative Commons Public Domain Dedication waiver (http://creativecommons.org/publicdomain/zero/1.0/) applies to the data made available in this article, unless otherwise stated. 


\section{Background}

Listeria monocytogenes is a foodborne pathogenic bacterium responsible for listeriosis, a non-invasive (i.e. febrile gastroenteritis) or invasive (i.e. meningitis and bacteraemia) disease with a statistically significant increasing trend of confirmed human cases in Europe (EU) during the last ten years [1]. Notwithstanding the much lower incidence of infections caused by L. monocytogenes $(0.48$ cases per 100,000 population in 2017) in comparison to Campylobacter and Salmonella enterica, the high fatality rate $(13.8 \%)$ of listeriosis (1633 human cases) in the EU in 2017 remains a serious concern for public health authorities [1]. L. monocytogenes is mainly transmitted to humans by ingestion of contaminated food, especially RTE food products such as dairy, meat and fish products [2]. The highest percentage of L. monocytogenes noncompliance in RTE foods at processing sites was reported in RTE fishery products (3-10\%) over 7 years (2008-2015) monitoring [2]. Contamination of RTE products is often linked to the occurrence of strains able to colonize harbourage sites and persist after cleaning and disinfection (C\&D) in FPEs [3]. So far there is no full agreement in the scientific community on the definition of L. monocytogenes persistence, however, the repeated isolation over a long period of the same subtype from the same FPE has been proposed [3, 4]. Indeed, specific subtypes may persist and/or be reintroduced at different times within FPEs. The persistence of certain subtypes of $L$. monocytogenes in food processing facilities or on equipment has been reported for up to 10 years and linked to food contamination from farm to fork [5]. The persistence of such strains in the food system has also been observed to play a significant role in listeriosis outbreaks during the last decades [6-8].

The establishment of persistent $L$. monocytogenes subtypes in a specific FPE may occur due to introduction(s) of resistant strain(s), and/or adaptation to the selection pressure met in the FPEs. In addition, the adaptation to particular environmental niches may shelter them from sanitizers used in the food industry. Persistence of such subtypes in RTE production premises is most likely promoted by i) food safety noncompliance (e.g. improper hygiene condition, failure to clean and disinfect food equipment, inadequate food facilities, etc.), ii) possible reintroductions of genotypes showing persistence potentials from external habitats or raw materials, iii) recontamination events due to inadequate sanitisation, and iv) the promoted survival and multiplication under suboptimal conditions in environmental niches [9, 10]. All these factors (either individually or in combination) along with the dynamics of L. monocytogenes populations and the complexity of the transmission pathways of persistent and transient strains in FPEs make the identification of the point of exposure source a critical task in risk management, public health preventions and food industry interventions.

The circulation of different L. monocytogenes subtypes was observed in food environments and clinical samples [11]. Strains from L. monocytogenes lineage II and serotype $1 / 2$ a have been more frequently collected in foods and food processing environments than strains from lineage I [12]. Accordingly, L. monocytogenes clonal complexes (CCs), defined as clusters of multilocus sequence typing (MLST) that share at least six alleles, have been epidemiologically associated with human listeriosis and with foods, based on the relative frequency among clinical and food-related sources [12-14]. These observations suggest that some $L$. monocytogenes clonal groups might harbour unique genotypic and phenotypic features facilitating their survival and growth in food and FPEs, as well as their potential transmission to humans. Multiple plasmid-borne homologous genes have been recently associated with lineage II food isolates [15]. Without a full description of the causal mechanisms promoting this particular phenotype, strains belonging to food-associated clonal groups of lineage II, including CC121, CC9, CC8, CC101, CC7 and CC204, have been shown to be persistent in FPEs [16-22].

Biofilm-forming ability, physiological adaptation and tolerance to environmental stresses such as temperature, osmotic and oxidative stresses as well as resistance to heavy metals and disinfectants have been phenotypically and genotypically investigated in specific serotypes and CCs of $L$. monocytogenes to elucidate their persistence mechanisms [19, 23-29] and comprehensively reviewed by Bergholz et al. (2018) [30]. Hence, subtype-specific genetic biomarkers contributing to the persistence phenotype have been described. So far Stress Survival Islet 1 (SSI-1) and SSI-2, including gene clusters involved in low $\mathrm{pH}$ and high salt concentrations tolerance as well as in alkaline and oxidative stress response, have been identified in L. monocytogenes predominantly belonging to serotypes $1 / 2 c, 3 b, 3 c$ and to CC121 [31-33]. A recent study of Hingston et al. (2017) associated the presence/absence and variations of genetic biomarkers such as the virulence gene inlA to different levels of cold and desiccation tolerance in specific serotypes of $L$. monocytogenes [25]. Tolerance to disinfectants based on quaternary ammonium compounds (QACs), such as benzalkonium chloride (BC), and heavy metals, such as cadmium $(\mathrm{Cd})$ and arsenic (As), have been described in persistent and presumably transient L. monocytogenes strains in FPEs [19, 23, 27, 28, 34, 35], as well as linked to specific molecular mechanisms. For instance, the three-gene cassette $b c r A B C$, located either on the chromosome or transposable units of $L$. monocytogenes strains, was described as conferring an increased resistance to BC, a widely used QAC disinfectant in the food 
industry in the past few decades [36]. The bcrABC cassette was detected in $L$. monocytogenes strains belonging to CC121, CC5, CC9, CC8, CC14 and CC204 [19, 21, 26] but not to $\mathrm{CC} 7, \mathrm{CC} 101$ and $\mathrm{CC} 155[18,26]$. In addition, the transposon Tn6188 carrying the transporter $\mathrm{QacH}$, and the transposon LGI1, carrying a small multidrug-resistant (SMR) efflux pump encoded by emrE, both responsible for enhanced $\mathrm{BC}$ tolerance, have been described as specific to $\mathrm{CC} 121$ and $\mathrm{CC} 8$ strains, respectively [35, 37, 38]. Recommended levels of QACs concentrations in the food industry are much higher than resistance levels conferred by these genes [39] and highly effective against the growth of planktonic bacteria [40]. However, the ability to grow in complex surfaceassociated communities in the form of biofilms has been shown to play a role in the protection of certain $L$. monocytogenes strains [35, 41] and CCs (e.g. CC121, CC9, CC204) [20] on food processing plant surfaces, enhancing cells persistence potentials in the food industry. Several Cd-resistance determinants were described in $L$. monocytogenes chromosome (e.g. cadA3) and plasmids (e.g. cadA1 and cadA2) [42, 43], often together with putative copper resistance determinants and co-selected with QACs resistance efflux pump, as the case of a cadA2-harbouring plasmid [44]. The recently described cadA4 determinant [29] has been identified in the Asresistance island LGI2 of L. monocytogenes human strain Scott A and other L. monocytogenes serotype $4 \mathrm{~b}$ as well as in few persistent strains belonging to $\mathrm{CC} 14$ and CC204 of Lineage II $[17,19,45]$.

As results of these studies, the persistence of certain $L$. monocytogenes subtypes is more commonly considered to arise from a complex combination of several factors rather than a single genetic or individual trait $[3,23,46]$. Hence, understanding the genome-wide diversity and dynamics of MGEs in relevant L. monocytogenes CCs for the food industry is crucial to gain useful information to disentangle their persistence in FPEs. Based on whole genome sequencing (WGS), this can be currently achieved by investigating single nucleotide polymorphisms (SNPs) at the core level (i.e. genomic sequences conserved across the whole population) and the presence/absence of genes at the accessory level (i.e. genes only present in subgroups of the population). Furthermore, investigating accessory genes enriched in strains under particular environmental conditions may help to unravel their adaptation mechanisms. The purpose of this study was, therefore, to explore the genomic diversity across L. monocytogenes subtypes repeatedly isolated for 2 up to 6 years from food and FPEs samples collected in three seafood processing facilities closely located in the French region of Boulogne-sur-Mer. The main aims were to determine highly-related persistent clonal strains and identifying genetic biomarkers that can be used to predict their adaptation and long-term survival in food-processing facilities, using a combination of de novo whole-genome analyses.

\section{Results \\ Distribution of CCs in persisting L. monocytogenes isolates from three French seafood facilities}

A comparative genomics analysis was performed in this study based on a selection of $94 \mathrm{~L}$. monocytogenes isolates belonging to the prevalent pulsotypes repeatedly collected over time from food and FPEs of the three different French RTE seafood processing plants (A, B, C) (Additional file 1).

In accordance with previous studies $[3,4,46]$, the selected $L$. monocytogenes genotypes (i.e. multiple isolates with indistinguishable PFGE profiles) were considered as putatively persistent since isolated over 6 months from the same or different source (e.g. RTE products and FPEs) within the same facility. The 94 food and FPEs isolates of $L$. monocytogenes CCs were recurrently collected over 2 to 6 years from smoked-herring and smoked-salmon producing plant A $(n=35)$ and B $(n=41)$, as well as shrimp processing plant $\mathrm{C}(n=18)$. After reads processing through ARTwork [47], two isolates out of the 96 sequenced were excluded because of suspected contaminations (Additional files 2 and 4). An overview of the draft genomes quality, i.e. genome length, N50 values, GC content and number of genes CDSs, including mapping parameters, i.e. depth and breadth of reads coverage, of the $94 \mathrm{~L}$. monocytogenes isolates is reported in Additional file 3. In accordance with the typical range previously described for L. monocytogenes genomes [17, 48-50], the genome size ranged between 2.92 and $3.36 \mathrm{Mbp}$ with a GC content of $37.8 \%$. The number of coding genes (i.e. coding DNA sequences; CDSs) varied from 2810 in the smallest genomes to 3100 in the largest ones. Genomes size variation appear most likely impacted by the presence/absence of accessory genetic elements like plasmids, prophages and transposons harboured by $\sim 70 \%, \sim 56 \%$ and $\sim 51 \%$ of isolates, respectively. Based on the 7-loci PubMLST schema, different $L$. monocytogenes CCs were found to co-exist into the different seafood processing plants (Fig. 1). Isolates collected over at least 15 months from plant A were classified as CC7 $(n=13)$, CC121 $(n=11)$ and CC204 $(n=11)$, whereas isolates collected over nearly 6 years from plant B were assigned to CC101 $(n=14)$, CC155 $(n=14)$ and CC204 $(n=13)$. In contrast, the 18 isolates from the shrimp producing plant $C$ belonged only to $\mathrm{CC} 121$ and were isolated during a 4-year sampling period (2008-2012) different than the time of isolation in plant A (1998-2001) and B (1998-2004).

\section{Phylogenomic clustering and pairwise SNP differences of phylogroups revealed intra- and inter-plant persisting clones}

To untangle the genetic relationships of the $94 \mathrm{~L}$. monocytogenes strains belonging to different CCs but isolated 
Tree scale: 0.001

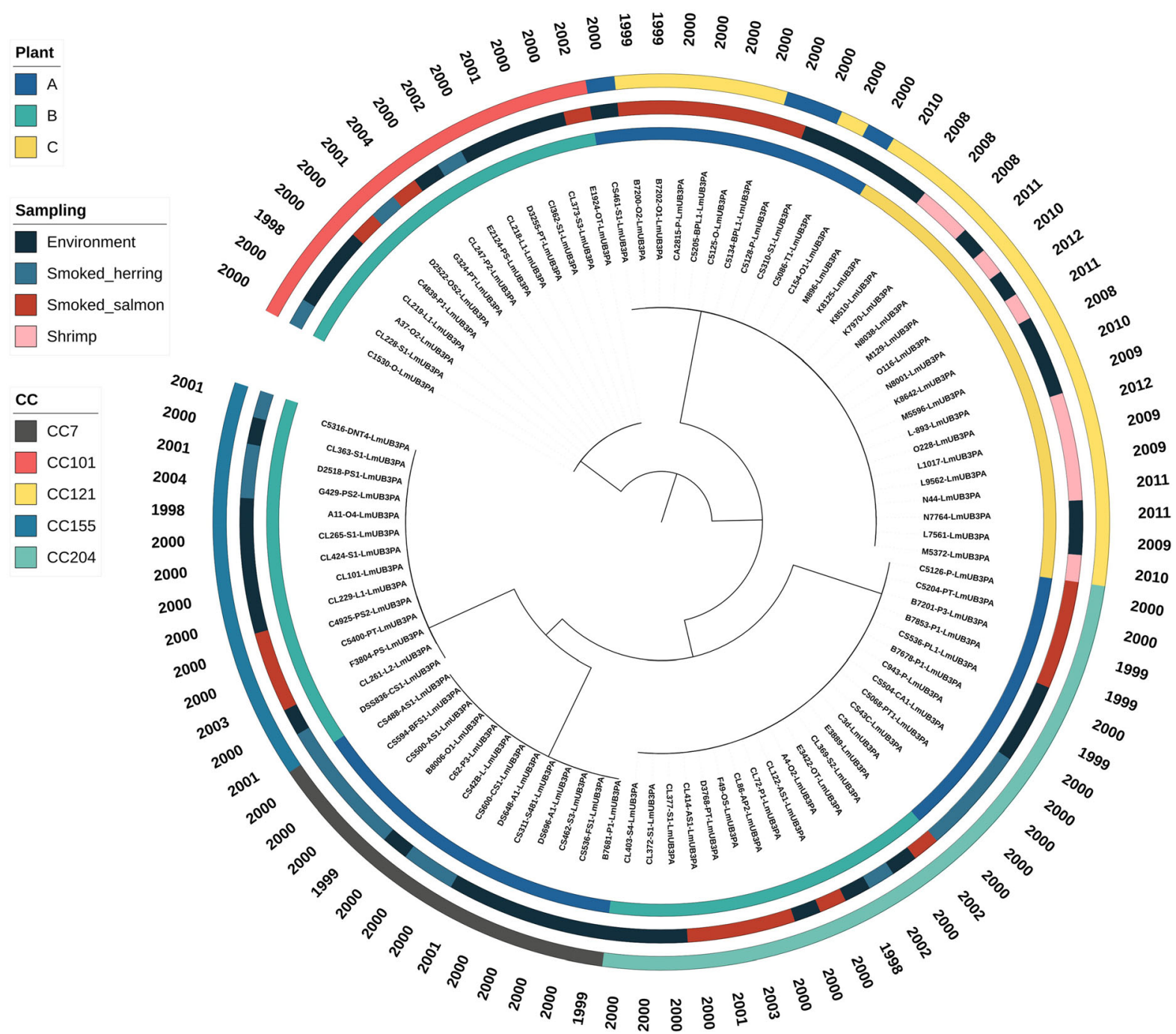

Fig. 1 SNPs-based phylogenomic reconstruction. The phylogenetic structure of the five L. monocytogenes CCs from the food processing plants was visualized on iTOL (https://itol.embl.de/). The maximum-likelihood phylogenomic reconstruction is based on 50,349 core genome SNPS extracted using iVARCall2 from 94 genomes. The originating processing plant, source, CC and date of isolation are shown on the tree (from inner to outer circles)

in the same environment for a long timeframe (over 26 years), an assembly-free core genome SNPs-based strategy was applied [51]. The Maximum-Likelihood (ML) phylogenomic tree was built on the concatenated core genome variants consisting of 50,349 SNPs identified among the 94 genomes (Fig. 1). Overall, the core genome SNPs-based phylogenomic reconstruction showed that the clusters of genomes correspond to the CC-types rather than with the sources or years of isolation (Fig. 1).

Albeit the isolation time spanned several months or years, the pairwise SNP distances between the isolates from individual CCs (intra-CC) were limited (highest mean 30 SNPs, range 0-63). In contrast, expected high pairwise SNP differences (ranging from 20,451 to 25, 534) were observed between CCs (inter-CC) (Fig. 2). The highest genetic diversity was detected in CC121 (i.e. 1st and 3rd quartiles of $\log _{10}$ pairwise SNP distances = 0.69 and 1.7, corresponding to 5 and 51 SNPs, respectively). Nevertheless, considering the CC121 strains from plant A and B separately, much lower levels of pairwise SNP differences were observed. For instance, 10 out of 11 isolates from plant A were characterized by pairwise distances ranging from 2 to 25 SNPs while the pairwise difference of the remaining isolate (CS461-S1LmUB3PA) ranged from 28 to 61 SNPs. Moreover, the 18 isolates collected over 4 years from plant $\mathrm{C}$ were remarkably genetically close with an overall pairwise SNP 


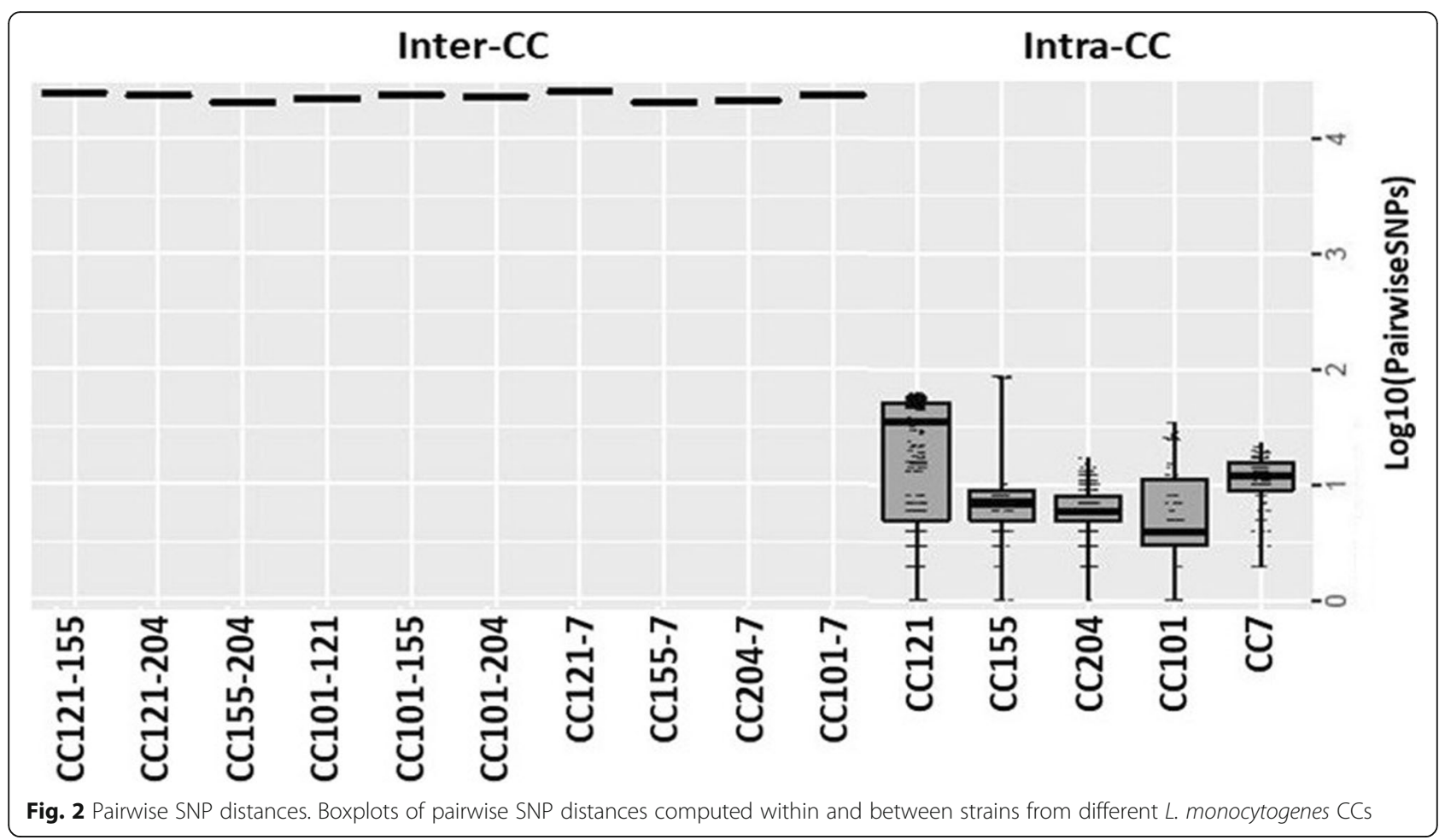

difference from 0 to 8 . A similar pairwise difference (0-10 SNPs) was found between CC155 isolates collected from plant B during 6 years (1998-2004), with the only exception for the DSS836-CS1-LmUB3PA strain showing a median pairwise distance of 86 SNPs. Likewise, the pairwise SNP distances between 12 out of 14 CC101 isolates collected from the same processing plant in a 4-years period (2000-2004) was very low (0-8). Interestingly, no SNP differences were observed between four of these isolates collected from different food matrices (i.e. smokedsalmon and smoked-herring) and the FPEs of plant B. The most genetically distant strains within CC101 were A37-O2-LmUB3PA and C1530-O-LmUB3PA, collected in 1988 and 2000, with a maximum pairwise difference of 15 and 34 SNPs, respectively. The genetic variation of $\mathrm{CC} 204$ isolates was likewise limited (0 to 17 SNPs), with a maximum of 10 SNPs between strains collected over 5 years (1998-2003) from plant B. Interestingly, less than 10 SNPs were also detected between CC204 isolates collected years apart in plant A and B from RTE foods and FPEs samples. Slightly higher levels of genetic diversity were found between $\mathrm{CC} 7$ isolates in comparison to the other clonal groups with the 1st and 3rd quartiles of 0.9 and 1.19, respectively (Fig. 2). However, these isolates were collected over several months in processing plant $\mathrm{A}$ and showed a maximum pairwise distance of 23 SNPs.
Pangenomic extraction and clustering of accessory genes reveal CC-specific genetic traits

Given the highly conserved core genome, a pangenome analysis was performed on the 94 genomes to investigate the genetic dynamics in terms of locus content within and between the phylogenetically shaped CCs. The pangenome was extracted with Roary [52] from the Prokkaannotated GFF3 files [53] and consisted of a matrix of 4935 group of orthologues of which 2526 core genes (i.e. genes shared by the $99 \%$ of isolates). The 2409 accessory genes included 29 soft-core genes (i.e. genes in $95 \% \leq$ strains < 99\%), 976 shell genes (i.e. genes in $15 \% \leq$ strains $<95 \%$ ) and 1404 cloud genes (i.e. genes in $0 \% \leq$ strains < $15 \%)$. These observations are consistent with previous comparative genomics studies unravelling the L. monocytogenes pangenome $[48,49,54,55]$. From the Phandango interactive visualization of the accessory genes based distance tree, associated metadata and the pangenome matrix (Additional file 6), five major clusters representing the CCs of $L$. monocytogenes genomes were identified. As observed in the SNP-based phylogenomic reconstruction, strains gathered within each cluster independently of the source or year of isolation. Most of $L$. monocytogenes genomes are conserved between CCs, however, a cluster distribution of CC-specific accessory traits was observed (Additional file 6). A preliminary screening of these accessory traits showed MGEs possibly explaining the successful adaptation of specific subclones to FPEs. For instance, putative prophage- and 
transposon-related clusters of genes were identified in the accessory matrix and appeared to be the major loci of genetic diversity across different CCs. Moreover, the intra-CC variations of the accessory genes content discriminated with higher resolution the genetic diversity observed in the core genome SNPs (Additional file 6). Gene clusters playing an important role in the survival of L. monocytogenes cells under the suboptimal conditions encountered in FPEs were detected in individual $\mathrm{CC}$ but also shared between different CCs. For instance, a transposon harbouring $\mathrm{Cd}$ - and As-resistance cassettes was conserved in all CC204 isolates from this study and inserted in the $y f b R$ gene. This genomic island showed high homology ( $99 \%$ average nucleotide identity (ANI)) to TnyfbR, a large $(\sim 35.7 \mathrm{kbp})$ transposon inserted in the $y f b R$ gene and recently identified in CC204 strains isolated from processing environment [17], as well as to the Listeria genomic island 2 (LGI2), previously described in the L. monocytogenes ScottA outbreak strain [45]. It contains the cadmium-resistance gene cadA4, conferring resistance to $35 \mathrm{mg} / \mathrm{L}$ of $\mathrm{Cd}$ chloride [29], and an As-resistance cassette including multiple loci (i.e., $\operatorname{ars} A-1$, $\operatorname{ars} A-2$, arsR, ars $D-1$, arsD-2, acr 3 ). Additional genetic features such as transcriptional factors and an FtsK domain protein are also included in this genomic island. All CC121 isolates from plant A and B harboured the Tn6188 transposon, a Tn554-like transposon already described in $\mathrm{CC} 121$ strains as responsible for enhanced tolerance to $\mathrm{BC}[38,56]$. This genetic island includes a TetR/AcrR family transcriptional regulator, three consecutive transposase genes $(\operatorname{tnp} A B C)$ and the $q a c C$ gene encoding for an SMR efflux pump involved in the extrusion of QACs [57]. The stress survival islet (SSI-1) [31] and the plasmidborne $b r c A B C$ efflux pump [24], respectively associated to acid/salt stress and to $\mathrm{BC}$ enhanced tolerance in FPE, were identified in genomes mainly from the FPEs and belonging to CC204, CC7 and in a subcluster of CC155. These observations suggest that persisting clones might acquire, conserve and possibly transfer specific MGEs as results of adaptive processes to FPE-associated stressors.
Contribution of plasmidome and mobile elements to FPEassociated stress survival

The plasmidome of the $94 \mathrm{~L}$. monocytogenes genomes were de novo reconstructed and the gene content investigated to untangle the MGEs contribution in the longterm survival of clonal groups. Combining plasmidSPAdes [58] and MOB-suite [59] algorithms, different plasmids were assembled and further compared with a comprehensive plasmid database (Table 1, Additional file 7).

A large $90.8 \mathrm{kbp}$ plasmid sequence (split into two contigs) was shared by $~ 92 \%$ of CC204 food and FPEs isolates $(n=22)$ from plant A and B, and $\sim 43 \%$ of CC155 $(n=6)$ isolates. Interestingly, all but one plasmidharbouring isolates from CC155 were from the FPEs of plant B. Although conserved across different CCs, the $90.8 \mathrm{kbp}$ plasmids were found almost identical (99.9\% ANI) in CC204 and CC155 strains collected in plant B from 2000 to 2003. On the other hand, the CC204 food and FPEs isolates from plant A were already plasmidpositive in the first sampling in 1999. A smaller sequence of $81.6 \mathrm{kbp}$ was reconstructed in a single contig across $~ 93 \%$ of food and FPEs isolates from CC7 $(n=$ 12). This plasmid was almost completely aligned with to the $90.8 \mathrm{kbp}$ plasmids showing high similarity $(99.9 \%$ ANI) over 99\% of the sequence. The plasmid from CC7 appears to be the result of a deletion of around 9 kbp sequence (Fig. 3), including oxidative stressresponse CDSs (e.g. qorB and RavA ATPase; see Additional file 8 for more details), and a recombination event with the plasmid from CC204 (Fig. 3). None of the plasmid sequences was found in two $\mathrm{CC} 7$ isolates from plant A as well as two CC204 isolates, one from each plant (A and B). All these isolates were collected at the beginning of the sampling timeframe (1998-1999). A total of 98 and 89 CDSs were predicted for the $90.8 \mathrm{kbp}$ and $81.6 \mathrm{kbp}$ plasmids, respectively, including genes implicated in stress response such as Cd-resistance determinants cadA2C and the efflux pump bcrABC conferring resistance to $\mathrm{BC}$ (Fig. 3).

One of the first plasmid harbouring the cadA2C/ bcr $A B C$ resistance markers was pLM80 from the $L$.

Table 1 In silico reconstruction and typing of the plasmidome and comparison with publicly available sequences from public databases NCBI and PLSDB. The plasmids reconstructed by plasmidSPAdes [58] and MOB-recon [59] are reported for each L. monocytogenes CC with predicted mobility based on the MOB-typer module [59]. Only the public sequences with ANI > 99.9\% and mash distance $<0.002$ were reported from NCBI and PLSDB databases [60], respectively

\begin{tabular}{llllll}
\hline CC & plasmidSPAdes & MOB-recon & MOB-typer & $\begin{array}{l}\text { NCBI } \\
(\text { ANI }>99.9 \%)\end{array}$ & $\begin{array}{l}\text { PLSDB } \\
\text { (mash dist < 0.002) }\end{array}$ \\
\hline 121 & $62.2^{\mathrm{a}}(22 ; 75 \%)^{\mathrm{b}}$ & $61.1(27 ; 93 \%)$ & conjugative & pLM6179 & pLM6179/pGMI16-004/pCFSAN022990 \\
7 & $81.6(12 ; 92 \%)$ & $81.6(12 ; 92 \%)$ & mobilizable & pLM80 & pCFSAN004330/ pLIS1/ pN1-011A/pCFSAN021445 \\
204 & $90.8(22 ; 92 \%)$ & $90.8(22 ; 92 \%)$ & mobilizable & pLM80 & pN1-011A/ pCFSAN021445/ pCFSAN004330/pLIS1 \\
155 & $90.8(6 ; 43 \%)$ & $90.8(6 ; 43 \%)$ & mobilizable & pLM80 & pN1-011A/ pCFSAN021445/ pCFSAN004330/pLIS1 \\
\hline
\end{tabular}

${ }^{a}$ Plasmid size in $k b p ;{ }^{b}$ Number of positive isolate and corresponding percentage in brackets 


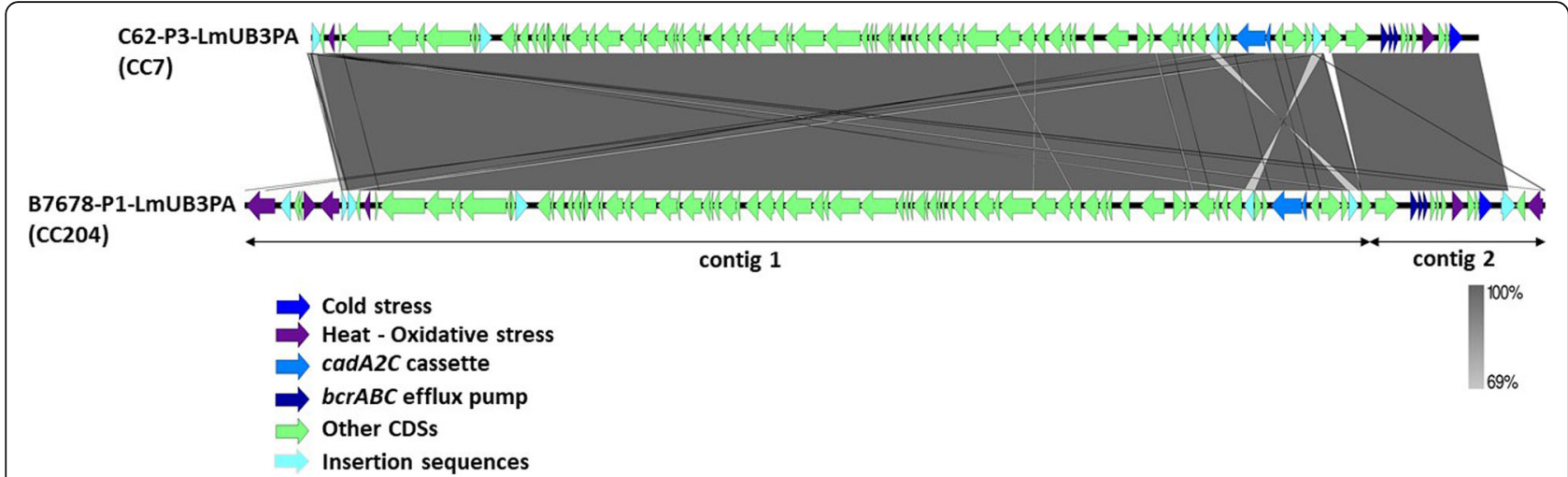

Fig. 3 Alignment of plasmid sequences from CC7 and CC204 strains. BLAST-based alignment of the closed $81.6 \mathrm{kbp}$ plasmid reconstructed from CC7 strain C62-P3-LmUB3PA and the 2 contigs $90.8 \mathrm{kbp}$ plasmid reconstructed from CC204 strain B7678-P1-LmUB3PA. Predicted CDSs are represented by arrows with CDSs relevant for L. monocytogenes stress response in FPEs coloured as in legend. Nucleotide identity levels (from 63 to $100 \%$ ) are indicated in grey shaded regions by colour intensity

monocytogenes strain H7858 responsible for the 19981999 hot dog outbreak [24]. High degrees of identity (> 99.9\% ANI over $\sim 96 \%$ coverage) were identified between plasmids from $\mathrm{CC} 7, \mathrm{CC} 204$ and $\mathrm{CC} 155$ plasmids and the $82.2 \mathrm{kbp}$ pLM80 (2 contigs: NZ AADR01000010, NZ_AADR01000058). Therefore, we considered them as pLM80-like plasmids. The pLM80like plasmids showed high identity (99.9\% ANI) also with highly conserved plasmids described in ST204 strains from food products and FPEs in Australia and Ireland [17], as well as in ST5 and ST204 strains from an Austrian cheese processing facility [21]. Interestingly, the time of sampling of these published genomes spans from 2000 to 2012.

A highly conserved $62.2 \mathrm{kbp}$ plasmid was also assembled in more than $75 \%(n=22)$ of CC121 genomes by plasmidSPAdes. This plasmid contained 61 predicted CDSs, which included Cd-resistance determinants cadA1C. The CC121 plasmids were closely related (ANI $>99.9 \%$; >99\% coverage) to a $62.2 \mathrm{kbp}$ plasmid named pLM6179 (acc. No. NZ_HG813250.1), previously described in L. monocytogenes strains persistent over 8 years (2000-2008) in an Irish farmhouse-cheese plant [22]. Therefore, we considered plasmids from CC121 as pLM6179-like plasmids. In a recent study comparing plasmid-cured strains and pLM6179-harbouring wild types, the latter showed higher tolerance levels in FPEassociated stressors [28], suggesting that the plasmidpositive strains may acquire survival advantages to temperature, osmotic, oxidative stress and disinfectants in FPEs.

Since similar plasmids were conserved within CCs as also spread across multiple CCs, the plasmids mobility was predicted with MOB-suite [59]. The pLM80-like plasmids were classified as "mobilizable" while the pLM6179-like plasmids as "conjugative". The MOB- reconstructions of plasmids from the draft genomes (i.e. ARTwork assembly) were also performed, retrieving the same number and type of plasmids in $\mathrm{CC} 7, \mathrm{CC} 155$ and CC204 genomes in comparison to plasmidSPAdes. Moreover, MOB-suite reconstructed plasmids from five additional draft genomes of CC121 (total $n=27$ ), in comparison to plasmidSPAdes. Exploring the plasmid sequences synteny in the additional positive genomes we further observed that they were identical to the pLM6179-like plasmids but rather than being in a single contig, they were integrated into the chromosome at the pyrG locus. pyrG is a psychrophilic gene encoding a CTP synthetase previously flagged as functional marker for site-specific integration of plasmids [61]. Combining results from both tools, only 2 environmental strains out of 29 CC121 strains, isolated in 2000 and 2009 from plant $\mathrm{A}$ and $\mathrm{C}$ respectively, lacked any observed plasmid sequences.

Typical genetic determinants of Listeria plasmid group 2 , including genes involved in plasmid replication (e.g., repA), maintenance (e.g., parA and soj gene) and putative transfer functions (e.g., type IV secretion system) [42] constituted the assembled plasmids. Genes conferring enhanced tolerance to processing plant-associated environmental stresses (e.g. cold, heat and osmotic) and virulence potential were also identified. See Additional file 8 for a detailed description of the main genetic components of plasmids.

The pLM80-like plasmids were genetically close (mash's distance $<1.77 \times 10^{-2}$ ) to different publicly available Listeria plasmids (Table 1) from PLSDB plasmid database [60]. In particular, a plasmid from $\mathrm{CC} 7$ was nearly identical (ANI >99\%; $100 \%$ coverage) to the 81.5 kbp pCFSAN004330 (acc. No. NZ_CP020834.1) and pLSI1 (acc. No. MH382833.1). The CC204 and CC155 plasmids showed higher similarity (ANI > 99\%; 
$100 \%$ coverage) to the $\sim 150 \mathrm{kbp}$ pN1-011A (acc. No. NC_ 022045.1) and pCFSAN021445 (acc. No. NZ_CP022021.1) in comparison to pCFSAN004330 and pLSI1 (91\% coverage) (Fig. 4 a). Except for pLSI1, all publicly available plasmids belonged to L. monocytogenes strains collected from food product and environment in the USA. In contrast, the pLSI1 plasmid belonged to the L. welshimeri strain 40/07 isolated in 2007 from sausage in Poland [62]. Korsak et al. (2019) described the L. welshimeri strain $40 / 07$ as $\mathrm{BC}$ - and Cdresistant, as well as the $b c r A B C / c a d A 2$-harbouring plasmids as potentially highly transferable [62]. These authors also defined the $\sim 150 \mathrm{kbp}$ pN1-011A and pCFSAN021445 as composite plasmids resulting from the recombination of a pLSI1-like plasmid and a small cadA1-harbouring plasmid similar to pl2015TE24968 from L. monocytogenes strain 2015 TE24968 (acc. No. CP015985).
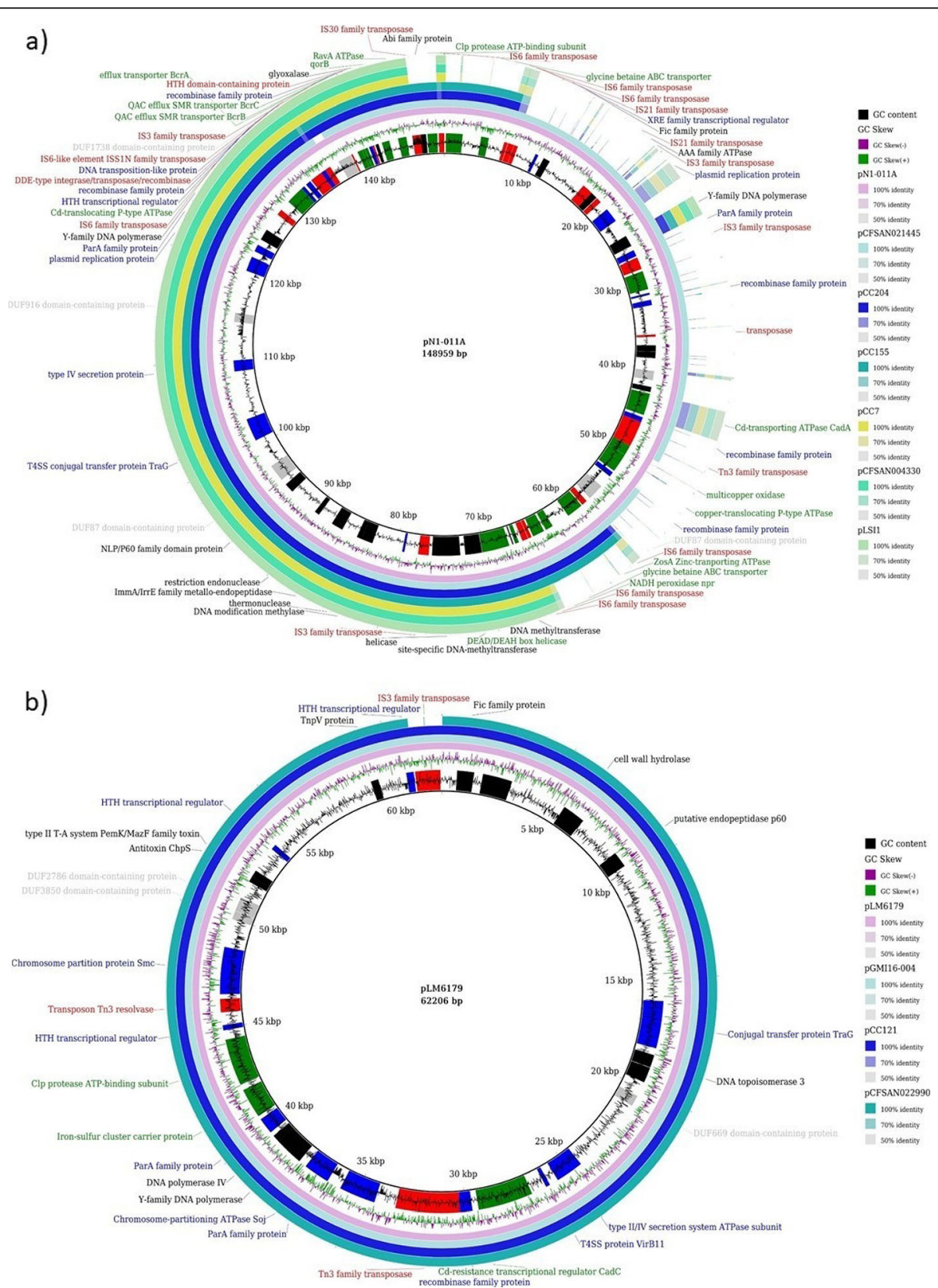

Fig. 4 BRIG comparison of the plasmids from this study and the public database PLSDB. Comparison of representative plasmids from CC7 (pCC7), CC155 (pCC155), CC204 (pCC204) (a) and CC121 (pCC121) (b) isolates with respective genetically closely related publicly available plasmids from PLSDB. The percentage of nucleotide identity refers to the comparison to the reference plasmid pN1-011A (a) and pLM6179 (b). Externally, CDS annotations are reported excluding hypothetical proteins and coloured based on functions (Additional file 8): transposase (red), stress response determinants (green), plasmid replication, maintenance and transfer (blue), and other functions (black) 
Two publicly available $L$. monocytogenes plasmids, the 62.2 kbp pGMI16-004 (acc. No. NZ_CP028184.1) and the $67 \mathrm{kbp}$ pCFSAN022990 (acc. No. NZ_CP020829.1), were reported as genetically close (mash distance < $2.41 \times 10^{-3}$ ) to the pLM6179-like plasmid from CC121 with a 99\% ANI and 99-97\% coverage. Interestingly, pGMI16-004 was found in the L. monocytogenes strain CFSAN054109 (GenBank acc. No. GCA_003030165.1) collected in Denmark from smoked fish in 2015.

\section{Unique prophage profiles characterize persisting clones}

The prophage $(\phi)$ profiles of the $94 L$. monocytogenes genomes were characterized using the PHASTER tool [63] for identification and annotation of putative prophage sequences and the integration loci. Prophages or phagederived elements were detected in all the L. monocytogenes genomes included in this study (Additional file 7). To deeply assess genetic diversity within clonal strains, presence and absence of questionable and intact regions, as well as conservation, ANI and integration site of the prophage sequences were considered. A total of 133 intact and questionable prophage regions were identified by PHASTER across 76 genomes from plant A and B. The 18 CC121 isolates from the shrimp-processing plant $\mathrm{C}$ harboured only incomplete prophage regions. The region size, phage components, insertion site, number of CDSs, name and percentage of the major phage organism, as well as the GC content were listed in Additional file 9. Besides the chimeric nature of these regions, a major Listeria phage organism (homologous to more than $50 \%$ of the total number of CDS of the region) was identified in the majority (>78\%) of the prophage dataset. The most commonly identified Listeria phages were: A600 [NC_009815], A118 [NC_003216], vB LmoS 188 [NC_028871] and LP-101 [NC_024387]. However, homologies to phages from other bacterial species such as phage 315.2 [NC_004585] from Streptococcus pyogenes were also reported (Additional file 9). PHASTER identified unique sets of prophages characterising strains from each clonal group, which presented from one (e.g. CC204, CC155) to three prophages per genome (e.g. CC7 and CC101) mostly integrated within tRNAs or $\operatorname{comK}$ genes (Table 2, Additional file 9). Within clonal groups, strains harboured highly conserved sequences in terms of number of identified regions and homologous CDSs, genes order and integration loci; although variants of the corresponding prophage length were observed (Table 2, Additional file 9). However, the alignment of these prophages showed high sequence similarity to each other (100\% ANI) (Additional file 10). The majority of prophages detected in the L. monocytogenes genomes were integrated into the chromosome and four insertion sites were identified: tRNA-Arg (ccg), tRNA-Arg (tct), tRNA-Ser (cga) and comK (Table 2).

The only exception was a small $22.1 \mathrm{kbp}$ phage homologous to vB LmoS 188, which was conserved as a single contig in all draft genomes from CC7. Interestingly, a high similarity (i.e. more than $92 \%$ ANI over $82 \%$ of sequence) was observed between this region and $\phi с о m K$ (i.e. a prophage mainly homologous to $\mathrm{vB} \operatorname{LmoS} 188$ ) in genomes from CC101 and CC155 (Additional file 9). Moreover, the alignment of $\phi \operatorname{comK}$ found in CC101, CC155 and CC204 showed high ANI values (above 94\%) over $\sim 70 \%$ of the sequences. Isolates from these CCs simultaneously occurred in plant B. Only one-third of the $\phi c o m K$ sequence conserved in genomes from CC7 (homologous to phage A118) was aligned to the $\phi c o m K$ sequence with an ANI higher than 95\% (Additional file 9). However, this sequence showed high nucleotide identity $(\sim 90 \%)$ for almost half of the $\phi$ tRNA-Arg (ccg) sequence (also homologous to phage A118) detected in genomes from CC121 isolated in the same plant (Additional file 9 and Additional file 10, Table 2). No similarity was detected between $\phi$ tRNA-Arg (ccg) and both tRNA-integrated prophages detected in all CC101 genomes. However, $\phi$ tRNA-Ser (cga) from CC101 showed high similarity (i.e. ANI >96.6\%; $84 \%$ coverage) to a фtRNA-Ser (cga) conserved in all but 3 genomes from CC7. These $\phi$ tRNA-Ser (cga) presented a highly mosaic structure with considerably lower similarity to Listeria phages in comparison to the other prophages and included CDSs homologous to Streptococcus phage 315.2 (Additional file 9). The remaining 3 genomes from CC7 harboured a longer version (73.0 kbp vs $33.2 \mathrm{kbp}$ ) of $\phi$ tRNA-Ser (cga) including around 50 additional phagerelated CDSs mainly homologous to Listeria LP-101 phage (Additional file 9). Interestingly, CS461-S1-

Table 2 Prophages and phage profiles of L. monocytogenes CCs gathered by integration hotspots

\begin{tabular}{|c|c|c|c|c|c|}
\hline & CC121 & CC7 & CC204 & CC101 & CC155 \\
\hline tRNA-Arg(ccg) & $49.9^{a}(10)^{b}$ & & & & \\
\hline tRNA-Arg(tct) & & & & $51.6(14)$ & \\
\hline tRNA-Ser(cga) & & $33.2(10) ; 73.0(3)$ & & $36.9(14)$ & \\
\hline comk & & $50.7(13)$ & $44.4(24)$ & 44.5 (14) & 46.8 (13) \\
\hline$-{ }^{c}$ & & $22.1(13)$ & & & \\
\hline
\end{tabular}

${ }^{a}$ Prophage size in $\mathrm{kbp} ;{ }^{b}$ Number of positive isolates, ${ }^{c}$ Extrachromosomal phage. 
LmUB3PA and DSS836-CS1-LmUB3PA genomes presented different prophage profiles in comparison to other isolates from persisting clones in plant A (i.e. CC121) and B (i.e. CC155), respectively, in addition to the higher pairwise distance (i.e. 28-38 SNPs and 83-86 SNPs, respectively). On the other hand, the C1530-OLmUB3PA genome presented the same prophage profile as the persisting clones from $\mathrm{CC} 101$ in plant $\mathrm{B}$, although the relatively high genetic distance (i.e. 25-34 SNPs).

\section{Pangenome-wide enrichment analysis identified several genetic elements associated with the plant}

Accessory gene clusters were statistically associated by Scoary [64] to identify genetic features enriched in clones persisting for long periods in a specific processing plant. Specificity, sensitivity and different $p$-values (e.g. Naïve, Bonferroni and Benjiamini-Hochberg (BH)) were calculated for each accessory gene and used to point out loci strongly associated with each plant (Additional file 11). With a $\mathrm{BH}$ value under $2 \times 10^{-5}, 513$ loci were almost exclusively present $(\sim 100 \%$ specificity $)$ in genomes from plant A $(n=204), \mathrm{B}(n=250)$ and C $(n=$ 59) (Additional file 11). Among these loci, a huge portion of hypothetical proteins $(n=298 ; 58 \%)$ was reported along with transposon- and prophage-related proteins originating from different bacterial species (L. monocytogenes, Bacillus, Yersinia) (Additional file 11). Most of these loci (97\%) were inherited together and located in different genomic segments while the remaining were spread across the genome. Interestingly several contiguous loci were included in different prophage regions identified in genomes from CC121 and CC7 isolated in plant A $(n=145), \mathrm{CC} 101$ and CC155 from plant B $(n=$ 91) as well as CC121 from plant C $(n=58)$ (Additional file 11). Even though the majority of plantassociated loci were CCs specific, 26, 18 and 15 phagerelated loci were shared by strains from different CCs simultaneously occurring in plant $\mathrm{A}, \mathrm{B}$ and $\mathrm{C}$, respectively. This is consistent with the similarities observed in the pairwise comparison of prophages described above.

Outside of the prophage regions, a cluster of 25 loci enriched in plant B was found to be part of a large (ca. $31.5 \mathrm{kbp}$ ) genomic island that we named Listeria genomic island 3 (LGI3). The LGI3 includes 29 predicted CDSs and chromosomally integrates the Cd-resistance determinants $\operatorname{cad} A 1 C$, flanked by the recombinase hin and the transposase Tn3 (Table 3) typical of pLM6179 plasmid. All the genomes containing the cadA1C-harbouring genomic island were members of CC101. In these strains, the genomic island is inserted upstream of the internalin gene inlJ (Lmo1413 homolog of strain EGD-e) and includes also genes putatively involved in DNA integration, conjugation, translocation and recombination (Fig. 5, Table 3). In comparison to the whole
NCBI database, only the $L$. monocytogenes reference strain ATCC 51775 (acc. No. CP025222.1), isolated from dairy products in Belgium in 1990-1992, showed sequence similarity (99.9\% of nucleotide identity) to LGI3. However, the ATCC 51775 sequence lacks the CadAC efflux cassette, suggesting that the $31.5 \mathrm{kbp}$ genetic island is specific to the CC101 clone from this study (Table 3).

An additional cluster of 26 contiguous loci involved in carbohydrate transport and metabolism was also statistically enriched in the CC101 persisting clone from plant B. This gene cluster included a number of CDSs specifically involved in the uptake, transport and utilization of primary carbon source (e.g. glucose, fructose, mannose and cellobiose) such as transketolase (tkt) and phosphotransferase system (PTS) operons (e.g. lic $A B C$ ). The correlation between the presence of these genes and the response of $L$. monocytogenes to environmental stress in FPEs still needs to be understood, however studies showing the up-regulation of PTS components in $L$. monocytogenes cells under exposure to QACs [65] as well as decreased transcript levels of PTS and related metabolic enzymes under salt stress [66] have been performed. An interesting 9-loci set enriched in plant A included a type I Restriction-Modification (RM) system that involves site-specific recombination within the gene $h s d S$ for the specificity subunit (S) and also on/off switches of gene expression $[67,68]$. The type I SRM complex is located between genes LMON_0301 and LMON_0303 in L. monocytogenes strain EGD (acc. No. HG421741), downstream to a recombinase locus (i.e. gene $L M O N_{-}$0299). This system has the potential to attack foreign DNA and other competitor RM systems as well as to kill the hosting cell through DNA breakage $[68,69]$. All strains belonging to CC7 harboured a type I SRM complex. A $\sim 10 \mathrm{kbp}$ genomic region, including a repeat region and clustered regularly interspaced short palindromic repeat (CRISPR)-Cas loci, was also identified as enriched in CC7 genomes and located downstream of a $\sim 24 \mathrm{kbp}$ incomplete phage sequence. The CRISPR-Cas loci was constituted by four cas genes (cas9, cas 1 , cas 2 and $\operatorname{csn} 2$ ) upstream of a $1820 \mathrm{bp}$ CRISPR array describing a Listeria type II CRISPR-Cas system. CRISPR-Cas loci encode for adaptive defence systems for protecting the bacterial cell from bacteriophage infection and further invading mobile DNA elements [70, 71].

\section{Discussions}

A combination of recently developed bioinformatics tools was applied in this study to assess the persistence phenotype through L. monocytogenes CCs contaminating food processing environments. The repeated introduction of the same subtypes in FPEs and/or the ability of 
Table 3 Gene content of LGI3 from nucleotide position 1,653,803 to 1,685,260 of CC101 strain A37-02-LmUB3PA. Loci are reported with Prokka annotation and homologous "locus tag" from L. monocytogenes reference strains ATCC 51775 for the genes enriched in food the plant (yes) and from L. monocytogenes 6179 for the non-enriched ones (no)

\begin{tabular}{|c|c|c|c|}
\hline Roary gene name & Prokka annotation & Reference strains locus tag & Enriched in food plant \\
\hline group_1188 & Hypothetical protein & CXL08_07550 & yes \\
\hline ydjM & Inner membrane protein YdjM & CXL08_07545 & yes \\
\hline group_1190 & Hypothetical protein & CXL08_07540 & yes \\
\hline group_1191 & Cna protein B-type domain protein & CXL08_07535 & yes \\
\hline group_1192 & DUF961 domain-containing protein & CXL08_07530 & yes \\
\hline group_1193 & DUF961 domain-containing protein & CXL08_07525 & yes \\
\hline ftsk & DNA translocase FtsK & CXL08_07520 & yes \\
\hline group_1195 & Gram-positive cell wall anchor protein & CXL08_07515 & yes \\
\hline group_1196 & Replication initiation factor & CXL08_07510 & yes \\
\hline group_1197 & Hypothetical protein & CXL08_07505 & yes \\
\hline group_1198 & Hypothetical protein & CXL08_07500 & yes \\
\hline group_1199 & Hypothetical protein & CXL08_07500 & yes \\
\hline group_1200 & Hypothetical protein & CXL08_07495 & yes \\
\hline $\operatorname{ard} A$ & Antirestriction protein ArdA & CXL08_07495 & yes \\
\hline group_1202 & Conjugative transposon protein TcpC & CXL08_07480 & yes \\
\hline group_723 & Hypothetical protein & CXL08_07475 & yes \\
\hline group_1203 & TcpE family protein & CXL08_07475 & yes \\
\hline group_1204 & Putative conjugal transfer protein & CXL08_07470 & yes \\
\hline infB_1 & Translation initiation factor IF-2 & CXL08_07465 & yes \\
\hline mepM & Murein DD-endopeptidase MepM & CXL08_07460 & yes \\
\hline group_1207 & Hypothetical protein & CXL08_07455 & yes \\
\hline group_1208 & Tn3 transposase DDE domain protein & LM6179_p0036 & no \\
\hline hin & DNA-invertase hin & LM6179_p0035 & no \\
\hline $\mathrm{cadC}$ & Cd-resistance transcriptional regulatory protein CadC & LM6179_p0034 & no \\
\hline cadA1 & Putative Cd-transporting ATPase & LM6179_p0033 & no \\
\hline group_1212 & Hypothetical protein & CXL08_07450 & yes \\
\hline group_1213 & Helix-turn-helix domain DNA-binding protein & CXL08_07445 & yes \\
\hline xerC_1 & Site-specific tyrosine recombinase XerC & CXL08_07440 & yes \\
\hline group_1215 & Helix-turn-helix domain DNA-binding protein & CXL08_07435 & yes \\
\hline
\end{tabular}

certain subtypes to adapt and survive to multiple environmental stressors encountered in FPEs [3, 4] challenge the effort to unravel the genetic basis of $L$. monocytogenes persistence in food processing for decades. The persistence of strains in RTE food producing plants is also of greatest concern when clinically relevant genotypes contaminate the related FPEs. For instances, CC101 and CC155, highly associated with human clinical listeriosis cases [12], represent clonal groups with enhanced pathogenic potential in comparison to CC121 and CC204, mainly found in foods and FPEs [12]. The reasons why some clonal groups of $L$. monocytogenes are recurrently isolated in the FPEs is still under discussion, as well as the dynamics of genetic elements potentially enhancing their persistence. In the last decades, several studies aiming to determine the basis of persistence in RTE industry and to trace-back outbreaks-related $L$. monocytogenes strains have been performed based on conventional molecular typing [72-75]. However, the advantages provided by analyses of genome-scale data through comparative genomic approaches have been recently demonstrated in the context of $L$. monocytogenes strains persisting in FPEs [4, 16, 18, 19, 23, 76-78]. These studies also suggested differences in distribution and fitness of CCs in geographical regions and evolutionary niches. Identify and characterize the CCs recurring in specific processing plants, along with the role of genetic elements contributing to their adaptation to the FPEs stressors may therefore boost the assessment of the L. monocytogenes persistence phenotype [30]. We tested 


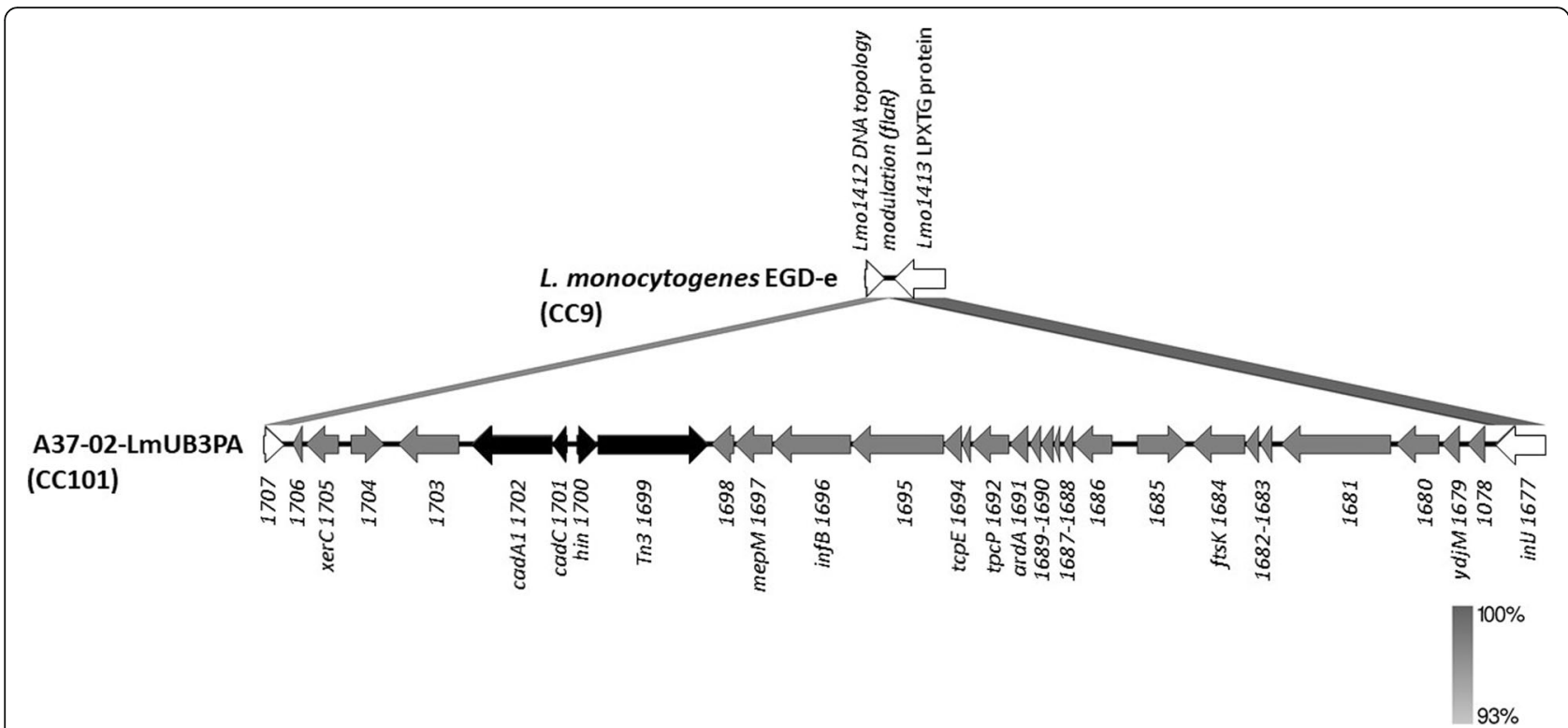

Fig. 5 Genomic organization of LGI3 of A37-02-LmUB3PA strain. Within LGI3, genes enriched in plant B and the integrated Cd-resistance cassette are shown by grey and black arrows, respectively, while LGI3-flanking genes in EGD-e strain are shown by white arrows. The Prokka locus tag is reported for each gene

this hypothesis focusing on the core and accessory genomes of $L$. monocytogenes subtypes repeatedly collected over 2-6 years from food and food-related environments of three closely located plants producing RTE seafood in France.

Persistent strains and newly introduced strains in the FPEs by raw material have been described in relation to specific STs or CCs $[18,20,78,79]$. With a similar objective, some authors $[18,78,80]$ proposed arbitrary thresholds ranging from 4 to less than 10 or 20 until 25 SNPs to identify persisting and "truly persisting" clones of $L$. monocytogenes. Other authors suggested that the isolation of the same $L$. monocytogenes clonal type (CT) at the same sampling point after $C \& D$ procedures for a much shorter sampling interval (i.e. less than one month) in comparison to this study was a sufficient parameter to consider $L$. monocytogenes strains as persistent [23]. Our results show that CCs recurring in plants A, B and C represented persisting and even "truly persisting" plant-specific clones including long-term persistent strains with less than 25 pairwise SNPs differences. As already observed in previous studies [18, 78, 79], the repeated detection of the genetically close or even indistinguishable strains from different foods and FPEs during the whole sampling period (2-6 years) bolstered the intra-plant clonality of $L$. monocytogenes strains included in the present study. The only exceptions were single strains for CC155 (DSS836-CS1-LmUB3PA), CC101 (C1530-O-LmUB3PA) and CC121 (CS461-S1LmUB3PA), considered as transient and likely reintroduced in the FPEs due to the high genetic differences from the persisting clones. Clonal groups of strains from CC121 and CC204 were detected across different plants. Nevertheless, CC121 isolates collected in plant A were genetically distant from the highly clonal isolates colonizing plant $C$ and collected 8 years apart (pairwise differences range: 44-63 SNPs). On the other hand, pairwise difference ranging between 4 and 13 SNPs were found comparing $\mathrm{CC} 204$ isolates from plant A and B, suggesting that these strains are from a unique clone that might have been transferred between these two facilities. Some authors concluded that identical or nearly identical (median 2 to 11 SNPs) L. monocytogenes strains can occur in different retail deli environments without any links of known transmission [76]. Although the present study confirms this observation, we could also speculate that geographically close companies producing similar foodstuff (e.g. plant A and B) may receive raw seafood from the same suppliers, which would constitute a common source of introduction for potentially persistent $L$. monocytogenes strains. In addition, the presence of the CC204 clone in plants A and B during overlapping sampling timeframe (1999-2001) would support the hypothesis of an inter-plant transmission L. monocytogenes strains. This finding is consistent with recent studies highlighting the presence of L. monocytogenes clones from ST8, ST9, ST101 and ST14 shared by different meatprocessing establishments $[16,78]$.

The occurrence of persistent intra- and inter-plant clones was corroborated by investigating the accessory genome. A specific repertoire of MGEs (i.e. plasmids, prophages and transposons) characterized strains from 
individual CC with a high degree of conservation. Previous authors also did similar observation comparing few subtypes of persistent $L$. monocytogenes strains from processing plants $[16,18,21,22]$. Our study confirmed this finding in a wider diversity of L. monocytogenes population showing that strains from additional CCs can acquire and conserve MGEs possibly enhancing their adaptation and survival in controlled FPEs.

Plasmids encoding genes conferring increased tolerance under multiple stress conditions related to food and FPEs were identified in the analysed $L$. monocytogenes from CC121, CC7, CC155 and CC204. These plasmids showed high similarity with globally spread publicly available plasmids harboured by persistent $L$. monocytogenes strains from ST121, ST5 and ST204 isolated in food products and related FPEs [17, 21, 22, 79]. Experimental evidence that such plasmids from L. monocytogenes ST121 and ST5 contribute to cells survival under stress condition met in FPEs have been recently proved [28]. Previous authors also suggested the importance of plasmids in the contribution to the survival of $L$. monocytogenes in food and FPEs [16, 17, 21, 22]. However, Naditz et al. (2019) were the first to show it at phenotypic and genotypic levels examining wild type and plasmid-cured strains [28]. In particular, these authors showed that pLM6179 and p4KSM plasmids from ST121 and ST5, respectively, play a key role in the enhanced capacity of $L$. monocytogenes to face some food processing environment-associated stresses like heat, $\mathrm{BC}$, oxidation and osmotic stress. Moreover, MuhteremUyar et al. (2018) observed that possible horizontal gene transfer (HGT) of the p4KSM plasmid (also referred as pLM80-like plasmid) took place from strains belonging to dominant ST5 to rare ST204, which later occurred in the same FPE and become the most dominant ST along with ST5. In our study, pLM80 prototypes highly similar to $\mathrm{p} 4 \mathrm{KSM}$ were identified in all but one persisting strains from CC204 in plants A and B. Interestingly, this plasmid was identical to a plasmid identified in 6 out of 14 strains from CC155 mainly isolated in FPEs of the plant B. Overall plasmid positive strains from CC204 and $\mathrm{CC} 155$ were found over three years in plant B either in FPEs or food samples. Due to the earlier occurrence and higher conservation of the pLM80-like plasmid from CC204 in comparison to CC155 strains, the plasmids have likely been transferred from CC204 to CC155 presumably involving strains colonising the same environmental niche in plant B. A different scenario can be hypothesised in plant A, where a closed smaller plasmid highly similar to plasmids from CC204 was conserved in all but two CC7 strains. The two strains lacking this plasmid were isolated at the beginning of the sampling. Comparison of plasmids from CC204 and CC7 might suggest that plasmids from $\mathrm{CC} 7$ might have arisen from recombination of the 2 contigs identified in plasmids from CC204, accompanied by a deletion of around 9 kbp (9 CDSs) pre- or post-transfer from CC204 to CC7. The deleted sequence included several neighbouring genes involved in stress response (e.g., npr, zosA, cplB, $g b u C$ ) along with three CDSs annotated as insertion sequence (Fig. 3, see Additional file 8). The presence of several insertion sequences points out that plasmids from CC204 are composite plasmids which integrated the genes conferring additional tolerance to plantassociated stresses. However, the high homology observed between plasmid variants from CC204 and CC7 may also suggest that a common ancestral plasmid underwent sequence shuffling and integration/deletion of additional environmental stress response genetic determinants, as also observed by Fox et al. (2016) comparing plasmids from CC204.

Adaptation to specific environmental niches in FPEs and short-term evolution of both distantly and closely related $L$. monocytogenes strains have been linked to the diversification of prophages [50, 79, 81, 82]. ST-specific prophages showing a high degree of conservation in FPEs have been described for strains from ST121 and ST8, while prophages from strains belonging to ST204 presented a lower degree of conservation [16, 17, 22]. Moreover, certain L. monocytogenes strains seem to be genetically more prone to be attacked by bacteriophages and transfer prophage genetic material [16]. With CC7 and CC101 showing the highest number of prophage regions per genome, all information herein shows that genetically related strains, isolated at different spots of FPEs over a long time frame, harbour highly conserved prophages in terms of number of identified region, insertion sites and CDSs composition. Interestingly, the same prophage profile characterizes all strains from CC204 in plant $\mathrm{A}$ and $\mathrm{B}$ while a different prophage profile was observed between strains from $\mathrm{CC} 121$ in plant A and C. This supports the hypothesis that phylogenetically shaped CC204 represent an inter-plant clone in contrast to $\mathrm{CC} 121$ which includes two intra-plant clones. In addition to the high pairwise SNPs distance, CS461-S1LmUB3PA and DSS836-CS1-LmUB3PA genomes presented different prophage profiles in comparison to the persisting strains from $\mathrm{CC} 121$ and $\mathrm{CC} 155$, respectively. These elements support the hypothesis that transient strains (i.e. not persistent) are probably reintroduced by raw material in the processing plants. On the other hand, C1530-O-LmUB3PA genome presented the same prophage repertoire of the CC101 strains, although its pairwise differences from the persisting clone exceeded 25 SNPs. The fact of harbouring the same prophages of persistent strains, including the $\phi \operatorname{com} K$ considered as a driving force of fitness advantages of $L$. monocytogenes in food production environments [82], suggests to also 
consider this strain as potentially persistent. This observation supports the hypothesis from Fagerlund et al. (2016) and Knudsen et al. (2017) stating that all isolates within an ST or CC present the potential to be or become persistent, especially when sharing the same environment $[15,18]$.

Previous studies evidenced that $\phi c o m K$ are genetic markers involved in the niche-adaptation and persistence of L. monocytogenes in meat FPEs, in addition to characterize the genotype diversity within ST (e.g. ST121). According to these studies, the $\phi c o m K$ genotypes can be associated to individual food processing operations and allow accurate tracking of persistent strains as well as the design of intervention strategies to reduce the plant contamination $[10,56]$. Highly conserved $\phi \operatorname{comK}$ were detected in all the persisting clones analysed in the present study. Small genetic elements, including CDSs associated with the originating plant, were shared between the $\phi c o m K$ from different CCs collected in the same FPEs, but also between tRNA-integrated prophages found in different FPEs (Additional file 10). This suggests that possible homologous and/or nonhomologous recombination events occurred between different $L$. monocytogenes CCs as also described in previous studies focusing on the persistence of $L$. monocytogenes [16, 76, 82]. However, prophage sequences integrated at the same locus in different strains strongly diverged between plant-specific persisting clones. Moreover, most of the genes significantly enriched in the specific plants were located in distinct prophages, suggesting that these regions include unique genetic markers possibly contributing to niche-specific adaptation of persisting clones.

A cluster of 25 loci significantly enriched in the smoked-fish plant B was found to be included in a novel $\sim 31.5 \mathrm{kbp}$ genomic island chromosomally integrating Cd-resistance determinants (cadA1C). This region, that we named Listeria genomic island 3 (LGI3), is highly conserved and specific to CC101 strains which persisted in plant B over 6 years. Genetic islands in L. monocytogenes have been described as MGEs constituted by active integrase for HGT and fitness conferring genes [50]. Although in this study we have no evidence on the HGT of LGI3, the presence of genes putatively involved in DNA integration, recombination and conjugation in this genetic island suggests the possibility of LGI3 transfer to other persisting clones.

\section{Conclusions}

The combination of different bioinformatics solutions evidenced intra- and inter-plant L. monocytogenes clones persisting over years in seafood products and processing environments of three French RTE-producing plants. In addition, it provided insights into the dynamics of stress tolerance-related genetic markers promoting the persistence of L. monocytogenes CCs in FPEs.

Genetic elements such as prophage loci and LGI3 constitute a genetic signal that may contribute to enhanced adaptation of L. monocytogenes persistent clones in FPEs as well as to the identification of their originating site. Functional annotation studies should be performed to fully evaluate the role of these genetic elements in the long-term survival of L. monocytogenes clones in food processing plants. However, focusing on genetic markers during surveillance studies can improve the trackingback of $L$. monocytogenes strains potentially involved in clinical cases. Moreover, the investigation of prophage profiles along with pairwise SNP distances of phylogenetically shaped CCs may provide increased discriminatory power in the identification of persisting clones and inter-plant transmissions.

Genomics studies for tracking the main routes of transmission of L. monocytogenes will contribute to define optimal risk mitigation strategies in RTE seafood producing processing plant and support food safety management system in fishery production. Results from these studies would also support stakeholders and risk manager especially as consumption of RTE seafood products increased in recent years. Our results extend evidences from other authors [17, 21, 25] emphasizing that almost identical plasmids conferring enhanced survival against FPE-associated stresses are globally spread on a wider population of phylogenetically distant clones, most likely due to the high selective pressure and availability of these genetic elements in FPEs. Constant environmental pressures met in FPEs promote the survival of L. monocytogenes population $\mathrm{s}$ and likely impact the genome evolution through HGT of MGEs, acquisition or loss of genes, and to a lesser extent by rare DNA recombination events or point mutations. Plasmid and prophage profiling might be relevant for implementing genomics in advanced predictive modelling of growth and survival of L. monocytogenes CCs in RTE food and processing plant environments.

\section{Methods}

Bacterial strains and seafood facilities characteristics

Keeping in mind the aim of this study, a selection of persistent L. monocytogenes subtypes was set up from a strain collection of the department of fishery products and aquaculture (Boulogne-sur-Mer), one site of the Laboratory for Food Safety in the French Agency for Food, Environmental and Occupational Health \& Safety (Anses). L. monocytogenes contaminated samples were repeatedly collected over 2-6 years from three geographically closed seafood facilities producing RTE foods (Additional file 1): two smoked-fish companies manufacturing smoked-salmon and smoked-herring (plants A 
and B), and a shrimp-processing company (plant C). Raw salmon and herring were originated from northern European aquaculture industry and captured in the Atlantic sea, respectively, while shrimps were imported from different countries (e.G. Madagascar and Vietnam). In the three seafood processing companies, the various working surfaces were mainly constituted of stainless steel, PVC or polyurethane, and the cleaning and disinfection operations $(C \& D)$ were performed by service providers with QACs and/or peroxide-based solutions. The isolation of bacterial strains from contaminated samples was originally performed according to standard method EN ISO 11290-1 and - 2 [83] and pulsed-field gel electrophoresis (PFGE) typing with ApaI and $A s c \mathrm{I}$ restriction enzymes was carried out as previously described by Midelet et al. (2007) (Additional file 2). Recurrent pulsotypes from different food products (i.e. shrimp, smoked-salmon and smoked-herring) in the processing line and related working environment (in direct contact and without any contact with foods) were found to be either common to multiple plants or specifically associated to individual plant. Therefore, a panel of 96 strains was selected for WGS according to i) the repeated isolation of indistinguishable pulsotypes in the same facilities over several years, ii) the PFGE profile common to plants A and B and iii) PFGE profiles specific to each plant.

\section{Genomic DNA extraction and whole genome sequencing} Genomic DNA of selected $L$. monocytogenes strains was extracted using the Wizard ${ }^{\oplus}$ Genomic DNA Purification Kit (Promega, France) according to manufacturer's instructions for Gram-positive bacteria. Nanodrop ${ }^{\circ}$ Spectrophotometer and $\mathrm{Qbit}^{\circ}$ fluorimeter were used to assess the quantity of the extracted gDNA. Global integrity of gDNA (200 ng) was assessed via horizontal agarose electrophoresis (Seakem GTG Agarose gel at $0.8 \%$ in TBE $1 \mathrm{X}$ gel) and the library of genomes were sequenced by paired-end reads (i.e. $2 \times 150 \mathrm{bp}$ ) at the 'Institut du Cerveau et de la Moëlle' (ICM, France, https://icm-institute. org/fr/) using Nextera XT DNA Library Prep Kit and Nextseq500 sequencing system (Illumina).

\section{De novo assembly and genome annotation}

An in-house developed pipeline called ARTwork (Assembly of Reads and Typing workflow) [47] was used for the generation of draft genome assemblies. Briefly, short paired-end reads are decompressed, the coverage of reads is estimated with BBmap [84] to exclude reads lower than 20X, and the others are normalized at 100X with Bbnorm (part of the BBMap v35.85 package; http:// sourceforge.net/projects/bbmap). Reads are then quality checked and a report is produced using FastQC v0.11.8 [85] before trimming of adapters and low-quality reads (i.e. minimal length of $50 \mathrm{bp}$ and quality score of 20) with Trimmomatic v0.38 [86] Contigs are therefore generated using the SPAdes v3.9 assembler [87] and the MinHash v2.0 estimation of Jaccard similarity index [88] is applied to identify the closely related reference genome from an internal database of high-quality complete reference genomes. Reference-based scaffolding and gap closure steps are then performed with MeDuSa [89] and GMcloser [90], respectively. Then, scaffolds shorter than $200 \mathrm{bp}$ are trimmed with Biopython and annotation is performed with Prokka v1.13.4 [53]. Finally, a qualitative evaluation of the assembly is then performed by computing various metrics with QUAST [91]. High-quality draft genomes of 94 L.monocytogenes strains were obtained with ARTwork pipeline (Additional file 3 and Additional file 4), whose source code is available at https://github.com/afelten-Anses/ARtWORK. FASTQC and QUAST metrics were combined by MultiQC [92].

\section{MLST-defined clonal complexes (CCs)}

The clonal complex (CC) of each draft genome was defined in silico by a 7-gene multilocus sequence typing (MLST) schema (i.e. abcZ, bglA, cat, dapE, dat, ldh and lhkA) [93, 94] using the open-source command line MLST software mlst v2.16.1.

\section{Core genome SNPs-based phylogenomic reconstruction}

A reference-based variant calling analysis was performed using the recently developed iVARCall2 pipeline [51]. Short reads were mapped against the complete reference genome of $L$. monocytogenes EGD-e (acc. No. NC003210) covering on average 2,789,940 bp ( 95\%). Briefly, after trimming of pair-end reads with Trimmomatic v0.38 (i.e. length $>50$ bases and $\mathrm{GC}>30$ ), reads are mapped with the Burrows-Wheeler Alignment tool (BWA) v0.7.17 [95] and sorted with Samtools v1.9. Any potential read duplicates are then marked and removed, and a realignment around InDels is performed with the Genome Analysis Toolkit (GATK) v3.7. In particular, the GATK HaplotypeCaller algorithm (which provides local assembly around variant regions) is used for SNPs/ InDels calling and filtering in accordance with the best practice [96] for the retaining of high-confidence variants. Finally, SNPs and InDels are stored by the iVARcCall2 workflow in genome Variant Call Format (gVCF) files for each sample, a single VCF file for the whole genome dataset, a single FASTA file of concatenated core variants and another single FASTA file of pseudogenomes. The pseudogenomes were used for the generation of a phylogenomic tree with the IQ-tree software v1.6.7 [97] using the MoledFinder fast model-selection option [98] with an ultrafast bootstrap (UFboot) approximation approach [99] of 1000 bootstrap replicates. The interand intra-cluster pairwise SNPs differences were 
calculated and plotted using the R packages "ggplot2", "dplyr" and "harrietr", with a script adapted from the $\mathrm{R}$ package harrietr (https://github.com/andersgs/harrietr). In order to support the robustness of the phylogenomic reconstruction, another core genome SNPs-based phylogenomic reconstruction was also performed using Snippy v4.0.2 (https://github.com/tseemann/snippy) implementing Freebayes v1.2.0 [100] as variants caller. The topology and clustering of both trees were compared with the $\mathrm{R}$ packages dendextend and phangorn (Additional file 5).

\section{Pangenomic profile}

The pangenome of the $94 \mathrm{~L}$. monocytogenes strains was defined using Roary v3.11.2 [52] with a 95\% BLASTP identity cut-off. Briefly, the Prokka-annotated genomes in general feature format 3 (GFF3) files were used as Roary input to extract the coding sequences. The latters are filtered (i.e. sequences with more than $5 \%$ of Ns or less than $120 \mathrm{bp}$ and/or without start/stop codons are removed) and clustered at different levels to finally compute a matrix of presence and absence of each gene in each isolate. Genes conserved in the $99 \%$ of the isolates will represent the "core genes" while the non-core genes (i.e. present in $<99 \%$ of genomes) will be considered as "accessory genes". The pangenome and a neighbourhood joining tree inferred based on the binary matrix of presence and absence of accessory genes produced by Roary were visualized on Phandango v1.3.0 (http://jameshadfield.github.io/phandango/\#/) along with associated metadata. This tool allowed to interactively screen the presence of genetic markers relevant for unravelling the persistence of L. monocytogenes in FPEs (e.g. plasmidand transposons-associated resistance determinants) and their variations between strains.

\section{Enrichment of genes from each plant}

Investigating the differences in genes composition can lead to a better understanding of key processes such as selection and adaptation. Therefore, a gene enrichment analysis was performed using Scoary v1.6.16 [64] to identify patterns of genes significantly overrepresented in isolates from each plant (A, B and C). The Roary matrix and a pre-defined trait representing the studied plant of each isolate were used as Scoary input. The collapse flag was used in the enrichment analysis to avoid multiple comparison corrections of identically distributed genes in the sample, such as plasmid and prophage genes, which would not add any information individually. Patterns of loci were considered strongly associated to plant $\mathrm{A}, \mathrm{B}$ and $\mathrm{C}$ if the Benjamini-Hochberg (BH)-corrected $p$-value was less than $2 \times 10^{-5}$ and the specificity $>99 \%$ (i.e. almost exclusively present in trait-positive isolates). Manual refinement of gene annotations and assessment of the synteny of associated loci were carried out through Artemis software [101] and searching homology with BLASTn tool from NCBI [102].

\section{Plasmidome reconstruction}

The plasmidome (i.e. total plasmid populations in a given environment) of the $94 \mathrm{~L}$. monocytogenes strains was de novo predicted using a combination of the bioinformatics tools plasmidSPAdes [58] and MOB-suite [59]. As first, the plasmidSPAdes algorithm was used with default settings for extracting and assembling of putative plasmids from short reads, mainly based on differential estimations of median coverage from chromosomal and plasmid sequences. Then, the recently developed MOB-suite software was used to predict the transmissibility of the plasmidSPAdes assembled sequences. Based on the presence of relaxase, mate-pair formation and oriT sequences, the plasmid is classified as "conjugative" (i.e. if it includes both a relaxase and a mate-pair formation marker), "mobilizable" (i.e. if it includes only a relaxase or an oriT) and "non-mobilizable" (i.e. if both a relaxase and an oriT are missing). Moreover, the MOB-recon algorithm was used to identify plasmidic contigs from the draft genomic assemblies generated by ARTwork in order to confirm the performance of plasmidSPAdes assembler. The BLAST-based MOB-recon tool uses markers from sequence databases of known replicons and relaxase in conjunction with a reference database of clustered plasmids provided by MOB-cluster [59]. Finally, the PLSDB web-resource [60], a comprehensive large-scale database including 13,789 (November 2018) bacterial plasmid complete sequences, was used for a large-scale comparative analysis to retrieves any plasmid records (and its meta-information) similar to the herein assembled plasmids. The PLSDB database was interrogated using Mash [88] (i.e. the command dist) with a maximal $p$-value and distance thresholds set to $1 \times 10^{-1}$. Plasmids genome map and comparison were visualized using EasyFig v2.2.3 [103] and BRIG v1.0 [104].

\section{Prediction of prophages}

In order to identify the putative prophage sequences harboured by the L. monocytogenes strains analysed in this study, the 94 draft genomes were submitted through the URL API to the PHASTER (PHAge Search Tool Enhanced Release) server [63]. This application scores prophage regions as "intact", "questionable" or "incomplete" based on several criteria such as the number of CDSs homologous to certain phages, the percentages of CDSs that match a certain phage (i.e. major potential phage), the size of the phage region and specific phagerelated keywords (e.g. tail, head, capsid, portal, terminase, integrase, etc). The functional annotations of CDSs 
homologous to known phages are also provided. Intact and questionable regions with sequence length over 20 kbp were considered for the prophage profiling of the genome dataset and the average nucleotide identity (ANI) computed for each genome pairwise using FastANI v2.0 (https://github.com/ParBLiSS/FastANI).

\section{Supplementary information}

Supplementary information accompanies this paper at https://doi.org/10. 1186/s12864-020-6544-X.

Additional file 1. List of $94 \mathrm{~L}$. monocytogenes isolates included in the present study, associated metadata and sequence accessions.

Additional file 2. Characterization of selected strains through pulsedfield gel electrophoresis.

Additional file 3. Overview of the genomes, CC, and quality parameters of de novo assembly and read mapping.

Additional file 4 Quality metrics of mapping (i.e. iVARcall2) and de novo assembly (i.e. ARTwork) from the studied L. monocytogenes genomes.

Additional file 5. Topology and distance comparison between phylogenomic reconstructions based on variant calling analyses from iVARCall2 and Snippy pipelines.

Additional file 6 Accessory genes-based Neighbour-joining clustering and pangenome matrix of $94 \mathrm{~L}$. monocytogenes isolates.

Additional file 7. List of plasmids, prophages and transposons.

Additional file 8. Gene content of pLM80-like and pLM6179-like plasmids.

Additional file 9. List of prophages per genome and insertion sites, size, number of CDS and annotation.

Additional file 10. Pairwise comparison matrix of prophage sequences. Additional file 11. List of genes enriched in the originating plant.

\section{Abbreviations \\ As: Arsenic; BH: Benjamini-Hochberg; CC: Clonal Complexes; Cd: Cadmium; C\&D: Cleaning and disinfection; CDS: Coding DNA sequence; EU: European Union; FPE: Food processing environment; GFF: general feature format; gVCF: genome variant call format; HGT: Horizontal gene transfer; LGI: Listeria genomic island; MGE: Mobile genetic element; ML: Maximum-Likelihood; MLST: multilocus sequence typing; NCBI: National Center for Biotechnology Information; NJ: Neighbour-joining; RTE: Ready-to-eat; SNP: Single nucleotide polymorphism; ST: Sequence type; tRNA: Transfer RNA; WGS: Whole Genome Sequencing}

\section{Acknowledgements}

We thanks the 'Institut du Cerveau et de la Moëlle' (ICM, France) for performing genomes sequencing. We would also like to acknowledge PierreEmmanuel Douarre for fruitful discussions on plasmid-related theories.

\section{Authors' contributions}

FP designed the study, performed the bioinformatics and comparative analysis and drafted the manuscript. TB performed strain selection, subtyping and DNA extraction, and contributed to the manuscript writing. NR participated in the design of the study and bioinformatics analyses and assisted in the drafting of the manuscript. LM set up computing resources and edited the manuscript. AF implemented the ARTwork pipeline and MongoDB database. MYM and AB participated in the coordination of the study and edited the manuscript. LG conceived the study, participated in its design and in the comparative analysis, and assisted in drafting the manuscript. GMB participated in the design of the study and strains selection and subtyping. All authors read and approved the final manuscript.

\section{Funding}

This study was funded by the European Union Horizon 2020 Research and Innovation Program: COMPARE (collaborative management platform for detection and analyses of [re-]emerging and foodborne outbreaks in Europe: grant number 643476). The funding body played no role in the design of the study and collection, analysis, and interpretation of data and in writing the manuscript.

\section{Availability of data and materials}

All the short pair-end reads of the 94 Listeria monocytogenes included in this study have been submitted to the European Nucleotide Archive (http:// www.ebi.ac.uk/ena) and are available under the study accession No: PRJEB30603.

\section{Ethics approval and consent to participate}

Not applicable.

\section{Consent for publication}

Not applicable.

\section{Competing interests}

The authors declare no financial and non-financial competing interest.

\section{Author details}

'ANSES, Laboratory for Food Safety, University Paris-Est, Maisons-Alfort, France. ${ }^{2}$ ANSES, Laboratory for Food Safety, Boulogne-sur-Mer, France. ${ }^{3}$ INRAE, MalAGE, University Paris-Saclay, Jouy-en-Josas, France.

Received: 28 June 2019 Accepted: 30 January 2020

Published online: 06 February 2020

\section{References}

1. European Food Safety Authority and European Centre for Disease Prevention and Control (EFSA and ECDC). The European Union summary report on trends and sources of zoonoses, zoonotic agents and food-borne outbreaks in 2017. EFSA J. 2018;16:e05500.

2. Ricci A, Allende A, Bolton D, Chemaly M, Davies R, Escámez PSF, et al. Listeria monocytogenes contamination of ready-to-eat foods and the risk for human health in the EU. EFSA J. 2018;16. https://doi.org/10.2903/.jefsa. 2018.5134.

3. Carpentier B, Cerf O. Review - persistence of Listeria monocytogenes in food industry equipment and premises. Int J Food Microbiol. 2011;145:1-8.

4. Ferreira V, Wiedmann M, Teixeira P, Stasiewicz MJ. Listeria monocytogenes persistence in food-associated environments: epidemiology, strain characteristics, and implications for public health. J Food Prot. 2014;77:150-70.

5. Zhu Q, Gooneratne R, Hussain MA. Listeria monocytogenes in fresh produce: outbreaks, Prevalence and Contamination Levels. Foods. 2017;6. https://doi.org/10.3390/foods6030021.

6. Angelo KM, Conrad AR, Saupe A, Dragoo H, West N, Sorenson A, et al. Multistate outbreak of <span class="italic" >Listeria monocytogenes</span> infections linked to whole apples used in commercially produced, prepackaged caramel apples: United States, 2014-2015. Epidemiology \&amp. Infect. 2017;145:848-56.

7. McCollum JT, Cronquist AB, Silk BJ, Jackson KA, O'Connor KA, Cosgrove S, et al. Multistate outbreak of listeriosis associated with cantaloupe. N Engl J Med. 2013;369:944-53.

8. Tham W, Ericsson H, Loncarevic S, Unnerstad H, Danielsson-Tham M-L. Lessons from an outbreak of listeriosis related to vacuum-packed gravad and cold-smoked fish. Int J Food Microbiol. 2000;62:173-5.

9. Larsen MH, Dalmasso M, Ingmer H, Langsrud S, Malakauskas M, Mader A, et al. Persistence of foodborne pathogens and their control in primary and secondary food production chains. Food Control. 2014;44:92-109.

10. Verghese B, Lok M, Wen J, Alessandria V, Chen Y, Kathariou S, et al. comK prophage junction fragments as markers for Listeria monocytogenes genotypes unique to individual meat and poultry processing plants and a model for rapid niche-specific adaptation, biofilm formation, and persistence. Appl Environ Microbiol. 2011;77:3279-92.

11. Orsi RH, den Bakker HC, Wiedmann M. Listeria monocytogenes lineages: genomics, evolution, ecology, and phenotypic characteristics. Int J Med Microbiol. 2011;301:79-96.

12. Maury MM, Tsai Y-H, Charlier C, Touchon M, Chenal-Francisque V, Leclerca A, et al. Uncovering Listeria monocytogenes hypervirulence by harnessing its biodiversity. Nat Genet. 2016;48:308-13. 
13. Maury MM, Bracq-Dieye $H$, Huang L, Vales $G$, Lavina $M$, Thouvenot $P$, et al. Hypervirulent Listeria monocytogenes clones' adaption to mammalian gut accounts for their association with dairy products. Nat Commun. 2019;10: 2488.

14. Painset A, Björkman JT, Kiil K, Guillier L, Mariet J-F, Félix B, et al. LiSEQ whole-genome sequencing of a cross-sectional survey of Listeria monocytogenes in ready-to-eat foods and human clinical cases in Europe. Microbial Genomics. 2019. https://doi.org/10.1099/mgen.0.000257.

15. Pirone-Davies C, Chen Y, Pightling A, Ryan G, Wang Y, Yao K, et al. Genes significantly associated with lineage $\|$ food isolates of Listeria monocytogenes. BMC Genomics. 2018;19:708.

16. Fagerlund A, Langsrud S, Schirmer BCT, Møretrø T, Heir E. Genome analysis of Listeria monocytogenes sequence Type 8 strains persisting in Salmon and Poultry processing environments and comparison with related strains. PLoS One. 2016;11:e0151117.

17. Fox EM, Allnutt T, Bradbury MI, Fanning S, Chandry PS. Comparative genomics of the Listeria monocytogenes ST204 subgroup. Front Microbiol. 2016;7. https://doi.org/10.3389/fmicb.2016.02057.

18. Knudsen GM, Nielsen JB, Marvig RL, Ng Y, Worning P, Westh $\mathrm{H}$, et al. Genome-wide-analyses of Listeria monocytogenes from food-processing plants reveal clonal diversity and date the emergence of persisting sequence types. Environ Microbiol Rep. 2017;9:428-40.

19. Pasquali F, Palma F, Guillier L, Lucchi A, De Cesare A, Manfreda G. Listeria monocytogenes sequence types 121 and 14 repeatedly isolated within one year of sampling in a rabbit meat processing plant: persistence and Ecophysiology. Front Microbiol. 2018;9. https://doi.org/10.3389/fmicb.2018.00596.

20. Stoller A, Stevens MJA, Stephan R, Guldimann C. Characteristics of Listeria Monocytogenes strains persisting in a meat processing facility over a 4-year period. Pathog. 2019;8:32.

21. Muhterem-Uyar M, Ciolacu L, Wagner K-H, Wagner M, Schmitz-Esser S, Stessl B. New aspects on Listeria monocytogenes ST5-ECVI predominance in a heavily contaminated cheese processing environment. Front Microbiol. 2018;9. https://doi.org/10.3389/fmicb.2018.00064.

22. Schmitz-Esser S, Müller A, Stessl B, Wagner M. Genomes of sequence type 121 Listeria monocytogenes strains harbor highly conserved plasmids and prophages. Front Microbiol. 2015;6. https://doi.org/10.3389/fmicb.2015. 00380.

23. Cherifi T, Carrillo C, Lambert D, Miniaï I, Quessy S, Larivière-Gauthier G, et al. Genomic characterization of Listeria monocytogenes isolates reveals that their persistence in a pig slaughterhouse is linked to the presence of benzalkonium chloride resistance genes. BMC Microbiol. 2018;18:220.

24. Elhanafi D, Dutta V, Kathariou S. Genetic characterization of plasmidassociated benzalkonium chloride resistance determinants in a Listeria monocytogenes strain from the 1998-1999 outbreak. Appl Environ Microbiol. 2010;76:8231-8.

25. Hingston P, Chen J, Dhillon BK, Laing C, Bertelli C, Gannon V, et al. Genotypes associated with Listeria monocytogenes isolates displaying impaired or enhanced tolerances to cold, salt, acid, or desiccation stress. Front Microbiol. 2017:8:369.

26. Meier AB, Guldimann C, Markkula A, Pöntinen A, Korkeala H, Tasara T. Comparative phenotypic and genotypic analysis of Swiss and Finnish Listeria monocytogenes isolates with respect to Benzalkonium chloride resistance. Front Microbiol. 2017;8. https://doi.org/10.3389/fmicb.2017.00397.

27. Mullapudi S, Siletzky RM, Kathariou S. Heavy-metal and benzalkonium chloride resistance of Listeria monocytogenes isolates from the environment of Turkey-processing plants. Appl Environ Microbiol. 2008;74: 1464-8.

28. Naditz AL, Dzieciol M, Wagner M, Schmitz-Esser S. Plasmids contribute to food processing environment-associated stress survival in three Listeria monocytogenes ST121, ST8, and ST5 strains. Int J Food Microbiol. 2019;299: 39-46.

29. Parsons $C$, Lee $S$, Jayeola $V$, Kathariou S. Novel cadmium resistance determinant in Listeria monocytogenes. Appl Environ Microbiol. 2017;83: e02580-16.

30. Bergholz TM, den Bakker HC, Katz LS, Silk BJ, Jackson KA, Kucerova Z, et al. Determination of evolutionary relationships of outbreak-associated Listeria monocytogenes strains of serotypes $1 / 2 \mathrm{a}$ and $1 / 2 \mathrm{~b}$ by whole-genome sequencing. Appl Environ Microbiol. 2016;82:928-38.

31. Ryan S, Begley M, Hill C, Gahan CGM. A five-gene stress survival islet (SSI-1) that contributes to the growth of Listeria monocytogenes in suboptimal conditions. J Appl Microbiol. 2010;109:984-95.
32. Hein I, Klinger S, Dooms M, Flekna G, StessI B, Leclercq A, et al. Stress survival islet 1 (SSI-1) survey in Listeria monocytogenes reveals an insert common to Listeria innocua in sequence Type $121 \mathrm{~L}$. monocytogenes strains. Appl Environ Microbiol. 2011;77:2169-73.

33. Harter E, Wagner EM, Zaiser A, Halecker S, Wagner M, Rychli K. Stress survival islet 2, predominantly present in Listeria monocytogenes strains of sequence Type 121, is involved in the alkaline and oxidative stress responses. Appl Environ Microbiol. 2017;83.

34. Ratani SS, Siletzky RM, Dutta V, Yildirim S, Osborne JA, Lin W, et al. Heavy metal and disinfectant resistance of Listeria monocytogenes from foods and food processing plants. Appl Environ Microbiol. 2012;78:6938-45.

35. Ortiz S, López-Alonso V, Rodríguez P, Martínez-Suárez JV. The connection between persistent, disinfectant-resistant Listeria monocytogenes strains from two geographically separate Iberian pork processing plants: evidence from comparative genome analysis. Appl Environ Microbiol. 2015;82:308-17.

36. Lawrence CA. Mechanism of action and neutralizing agents for surfaceactive materials upon microorganisms. Ann N Y Acad Sci. 1950;53:66-75.

37. Kovacevic J, Arguedas-Villa C, Wozniak A, Tasara T, Allen KJ. Examination of food chain-derived Listeria monocytogenes strains of different serotypes reveals considerable diversity in inlA genotypes, mutability, and adaptation to cold temperatures. Appl Environ Microbiol. 2013;79:1915-22.

38. Müller A, Rychli K, Muhterem-Uyar M, Zaiser A, Stessl B, Guinane CM, et al. Tn6188 - a novel transposon in Listeria monocytogenes responsible for tolerance to benzalkonium chloride. PLoS One. 2013;8:e76835.

39. Tezel U, Pavlostathis SG. Quaternary ammonium disinfectants: microbial adaptation, degradation and ecology. Curr Opin Biotechnol. 2015;33:296-304.

40. Coughlan LM, Cotter PD, Hill C, Alvarez-Ordóñez A. New weapons to fight old enemies: novel strategies for the (bio) control of bacterial biofilms in the food industry. Front Microbiol. 2016;7. https://doi.org/10.3389/fmicb. 2016.01641 .

41. Martínez-Suárez JV, Ortiz S, López-Alonso V. Potential impact of the resistance to quaternary ammonium disinfectants on the persistence of Listeria monocytogenes in food processing environments. Front Microbiol. 2016;7. https://doi.org/10.3389/fmicb.2016.00638.

42. Kuenne C, Voget S, Pischimarov J, Oehm S, Goesmann A, Daniel R, et al. Comparative analysis of plasmids in the genus Listeria. PLoS One. 2010;5: e12511.

43. Lebrun M, Audurier A, Cossart P. Plasmid-borne cadmium resistance genes in Listeria monocytogenes are present on Tn5422, a novel transposon closely related to Tn917. J Bacteriol. 1994;176:3049-61.

44. Parsons $\mathrm{C}$, Lee $\mathrm{S}$, Kathariou S. Heavy metal resistance determinants of the foodborne pathogen Listeria monocytogenes. Genes. 2019;10:11.

45. Lee S, Rakic-Martinez M, Graves LM, Ward TJ, Siletzky RM, Kathariou S. Genetic determinants for cadmium and arsenic resistance among Listeria monocytogenes serotype $4 \mathrm{~b}$ isolates from sporadic human Listeriosis patients. Appl Environ Microbiol. 2013;79:2471-6.

46. Castro $H$, Jaakkonen $A$, Hakkinen $M$, Korkeala $H$, Lindström M. Occurrence, persistence, and contamination routes of Listeria monocytogenes genotypes on three Finnish dairy cattle farms: a longitudinal study. Appl Environ Microbiol. 2018;84:e02000-17.

47. Felten A. Anses workflow for reads process. 2019. https://github.com/ afelten-Anses/ARtWORK.

48. den Bakker HC, Desjardins CA, Griggs AD, Peters JE, Zeng Q, Young SK, et al. Evolutionary dynamics of the accessory genome of Listeria monocytogenes. PLoS One. 2013;8:e67511.

49. den Bakker HC, Cummings CA, Ferreira V, Vatta P, Orsi RH, Degoricija L, et al. Comparative genomics of the bacterial genus Listeria: genome evolution is characterized by limited gene acquisition and limited gene loss. BMC Genomics. 2010;11:688.

50. Kuenne C, Billion A, Mraheil MA, Strittmatter A, Daniel R, Goesmann A, et al. Reassessment of the Listeria monocytogenespan-genome reveals dynamic integration hotspots and mobile genetic elements as major components of the accessory genome. BMC Genomics. 2013;14:47.

51. Felten A, Vila Nova M, Durimel K, Guillier L, Mistou M-Y, Radomski N. First gene-ontology enrichment analysis based on bacterial coregenome variants: insights into adaptations of Salmonella serovars to mammalianand avian-hosts. BMC Microbiol. 2017;17. https://doi.org/10.1186/s12866017-1132-1.

52. Page AJ, Cummins CA, Hunt M, Wong VK, Reuter S, Holden MTG, et al. Roary: rapid large-scale prokaryote pan genome analysis. Bioinformatics. 2015;31:3691-3. 
53. Seemann T. Prokka: rapid prokaryotic genome annotation. Bioinformatics. 2014;30:2068-9

54. Hilliard A, Leong D, O'Callaghan A, Culligan EP, Morgan CA, DeLappe N, et al. Genomic Characterization of Listeria monocytogenes Isolates Associated with Clinical Listeriosis and the Food Production Environment in Ireland. Genes (Basel). 2018;9. https://doi.org/10.3390/genes9030171.

55. Fritsch L, Felten A, Palma F, Mariet J-F, Radomski N, Mistou M-Y, et al. Insights from genome-wide approaches to identify variants associated to phenotypes at pan-genome scale: Application to $L$ monocytogenes' ability to grow in cold conditions. Int J Food Microbiol. 2019;291:181-8.

56. Rychli K, Wagner EM, Ciolacu L, Zaiser A, Tasara T, Wagner M, et al. Comparative genomics of human and non-human Listeria monocytogenes sequence type 121 strains. PLoS One. 2017;12:e0176857.

57. Piddock LJV. Clinically relevant chromosomally encoded multidrug resistance efflux pumps in Bacteria. Clin Microbiol Rev. 2006;19:382-402.

58. Antipov D, Hartwick N, Shen M, Raiko M, Lapidus A, Pevzner PA. plasmidSPAdes: assembling plasmids from whole genome sequencing data. Bioinformatics. 2016;32:3380-7.

59. Robertson J, Nash JHE. MOB-suite: software tools for clustering, reconstruction and typing of plasmids from draft assemblies. Microb Genom. 2018:4.

60. Galata V, Fehlmann T, Backes C, Keller A. PLSDB: a resource of complete bacterial plasmids. Nucleic Acids Res. 2019:47:D195-202.

61. Weidner G, d'Enfert C, Koch A, Mol PC, Brakhage AA. Development of a homologous transformation system for the human pathogenic fungus Aspergillus fumigatus based on the pyrG gene encoding orotidine 5' '-monophosphate decarboxylase. Curr Genet. 1998;33:378-85.

62. Korsak D, Chmielowska C, Szuplewska M, Bartosik D. Prevalence of plasmidborne benzalkonium chloride resistance cassette bcrABC and cadmium resistance cadA genes in nonpathogenic Listeria spp isolated from food and food-processing environments. Int J Food Microbiol. 2019;290:247-53.

63. Arndt D, Grant JR, Marcu A, Sajed T, Pon A, Liang Y, et al. PHASTER: a better, faster version of the PHAST phage search tool. Nucleic Acids Res. 2016;44: W16-21.

64. Brynildsrud O, Bohlin J, Scheffer L, Eldholm V. Rapid scoring of genes in microbial pan-genome-wide association studies with Scoary. Genome Biol. 2016;17:238.

65. Casey A, Fox EM, Schmitz-Esser S, Coffey A, McAuliffe O, Jordan K. Transcriptome analysis of Listeria monocytogenes exposed to biocide stress reveals a multi-system response involving cell wall synthesis, sugar uptake, and motility. Front Microbiol. 2014;5. https://doi.org/10.3389/fmicb.2014. 00068.

66. Bae D, Liu C, Zhang T, Jones M, Peterson SN, Wang C. Global gene expression of Listeria monocytogenes to salt stress. J Food Prot. 2012;75: 906-12.

67. Dybvig K, Sitaraman R, French CT. A family of phase-variable restriction enzymes with differing specificities generated by high-frequency gene rearrangements. Proc Natl Acad Sci U S A. 1998;95:13923-8.

68. Kobayashi I. Behavior of restriction-modification systems as selfish mobile elements and their impact on genome evolution. Nucleic Acids Res. 2001; 29:3742-56.

69. Murray NE, Type I. Restriction systems: sophisticated molecular machines (a legacy of Bertani and Weigle). Microbiol Mol Biol Rev. 2000;64:412-34.

70. Deveau H, Garneau JE, Moineau S. CRISPR/Cas system and its role in phagebacteria interactions. Annu Rev Microbiol. 2010;64:475-93.

71. Makarova KS, Wolf YI, Alkhnbashi OS, Costa F, Shah SA, Saunders SJ, et al. An updated evolutionary classification of CRISPR-Cas systems. Nat Rev Microbiol. 2015;13:722-36.

72. Lomonaco S, Decastelli L, Nucera D, Gallina S, Manila Bianchi D, Civera T. Listeria monocytogenes in Gorgonzola: subtypes, diversity and persistence over time. Int J Food Microbiol. 2009;128:516-20.

73. Mammina C, Parisi A, Guaita A, Aleo A, Bonura C, Nastasi A, et al. Enhanced surveillance of invasive listeriosis in the Lombardy region, Italy, in the years 2006-2010 reveals major clones and an increase in serotype 1/2a. BMC Infect Dis. 2013;13:152.

74. Norton DM, McCamey MA, Gall KL, Scarlett JM, Boor KJ, Wiedmann M. Molecular studies on the ecology of Listeria monocytogenes in the smoked fish processing industry. Appl Environ Microbiol. 2001;67:198-205.

75. Véghová A, Minarovičová J, Koreňová J, Drahovská H, Kaclíková E. Prevalence and tracing of persistent Listeria monocytogenes strains in meat processing facility production chain. J Food Saf. 2017;37.
76. Stasiewicz MJ, Oliver HF, Wiedmann M, den Bakker HC. Whole-genome sequencing allows for improved identification of persistent Listeria monocytogenes in food-associated environments. Appl Environ Microbiol. 2015:81:6024-37.

77. Palma F, Pasquali F, Lucchi A, Cesare AD, Manfreda G. Whole genome sequencing for typing and characterisation of Listeria monocytogenes isolated in a rabbit meat processing plant. Ital J Food Saf. 2017;6.

78. Morganti M, Scaltriti E, Cozzolino P, Bolzoni L, Casadei G, Pierantoni M, et al. Processing-dependent and clonal contamination patterns of Listeria monocytogenes in the cured ham food chain revealed by genetic analysis. Appl Environ Microbiol. 2016;82:822-31.

79. Harrand AS, Jagadeesan B, Baert L, Wiedmann M, Orsi RH. Evolution of Listeria monocytogenes in a food-processing plant involves limited single nucleotide substitutions, but considerable diversification by gain and loss of prophages. Appl Environ Microbiol. 2020. https://doi.org/10.1128/AEM.02493-19.

80. Nielsen EM, Björkman JT, Kiil K, Grant K, Dallman T, Painset A, et al. Closing gaps for performing a risk assessment on Listeria monocytogenes in readyto-eat (RTE) foods: activity 3, the comparison of isolates from different compartments along the food chain, and from humans using whole genome sequencing (WGS) analysis; 2017. https://doi.org/10.2903/sp.efsa. 2017.EN-1151.

81. Gilmour MW, Graham M, Van Domselaar G, Tyler S, Kent H, Trout-Yakel $\mathrm{KM}$, et al. High-throughput genome sequencing of two Listeria monocytogenes clinical isolates during a large foodborne outbreak. BMC Genomics. 2010;11:120.

82. Orsi RH, Borowsky ML, Lauer P, Young SK, Nusbaum C, Galagan JE, et al. Short-term genome evolution of Listeria monocytogenes in a noncontrolled environment. BMC Genomics. 2008:9:539.

83. Midelet-Bourdin G, Leleu G, Malle P. Evaluation of the international reference methods NF EN ISO 11290-1 and 11290-2 and an in-house method for the isolation of Listeria monocytogenes from retail seafood products in France. J Food Prot. 2007;70:891-900.

84. Bushnell B. BBMap: a fast, Accurate, Splice-Aware Aligner. 2014. https:// escholarship.org/uc/item/1h3515gn.

85. Andrews S. FastQC A Quality control tool for high throughput sequence data. 2010. http://www.bioinformatics.babraham.ac.uk/projects/fastac/.

86. Bolger AM, Lohse M, Usadel B. Trimmomatic: a flexible trimmer for Illumina sequence data. Bioinformatics. 2014;30:2114-20.

87. Bankevich A, Nurk S, Antipov D, Gurevich AA, Dvorkin M, Kulikov AS, et al. SPAdes: a new genome assembly algorithm and its applications to singlecell sequencing. J Comput Biol. 2012;19:455-77.

88. Ondov BD, Treangen TJ, Melsted P, Mallonee AB, Bergman NH, Koren S, et al. Mash: fast genome and metagenome distance estimation using MinHash. Genome Biol. 2016;17:132.

89. Bosi E, Donati B, Galardini M, Brunetti S, Sagot M-F, Lió P, et al. MeDuSa: a multi-draft based scaffolder. Bioinformatics. 2015;31:2443-51.

90. Kosugi S, Hirakawa H, Tabata S. GMcloser: closing gaps in assemblies accurately with a likelihood-based selection of contig or long-read alignments. Bioinformatics. 2015;31:3733-41.

91. Gurevich A, Saveliev V, Vyahhi N, Tesler G. QUAST: quality assessment tool for genome assemblies. Bioinformatics. 2013;29:1072-5.

92. Ewels P, Magnusson M, Lundin S, Käller M. MultiQC: summarize analysis results for multiple tools and samples in a single report. Bioinformatics. 2016;32:3047-8

93. Jolley KA, Maiden MC. BIGSdb: scalable analysis of bacterial genome variation at the population level. BMC Bioinformatics. 2010;11:595.

94. Ragon M, Wirth T, Hollandt F, Lavenir R, Lecuit M, Monnier AL, et al. A new perspective on Listeria monocytogenes evolution. PLoS Pathog. 2008;4: e1000146.

95. Li H, Durbin R. Fast and accurate short read alignment with burrowswheeler transform. Bioinformatics. 2009;25:1754-60.

96. der Auwera GAV, Carneiro MO, Hartl C, Poplin R, del Angel G, LevyMoonshine A, et al. From FastQ data to high-confidence variant calls: the genome analysis toolkit best practices pipeline. Curr Protoc Bioinformatics. 2013;43:11.10.1-11.10.33

97. Nguyen L-T, Schmidt HA, von Haeseler A, Minh BQ. IQ-TREE: a fast and effective stochastic algorithm for estimating maximum-likelihood phylogenies. Mol Biol Evol. 2015;32:268-74.

98. Kalyaanamoorthy S, Minh BQ, Wong TKF, von Haeseler A, Jermiin LS. ModelFinder: fast model selection for accurate phylogenetic estimates. Nat Methods. 2017;14:587-9. 
99. Minh BQ, Nguyen MAT, von Haeseler A. Ultrafast approximation for phylogenetic bootstrap. Mol Biol Evol. 2013;30:1188-95.

100. Garrison E, Marth G. Haplotype-based variant detection from short-read sequencing. arXiv:12073907 [q-bio]. 2012. http://arxiv.org/abs/1207.3907.

101. Carver T, Berriman M, Tivey A, Patel C, Böhme U, Barrell BG, et al. Artemis and ACT: viewing, annotating and comparing sequences stored in a relational database. Bioinformatics. 2008;24:2672-6.

102. Camacho C, Coulouris G, Avagyan V, Ma N, Papadopoulos J, Bealer K, et al. BLAST+: architecture and applications. BMC Bioinformatics. 2009;10:421.

103. Sullivan MJ, Petty NK, Beatson SA. Easyfig: a genome comparison visualizer. Bioinformatics. 2011;27:1009-10.

104. Alikhan N-F, Petty NK, Ben Zakour NL, Beatson SA. BLAST ring image generator (BRIG): simple prokaryote genome comparisons. BMC Genomics. 2011;12:402

\section{Publisher's Note}

Springer Nature remains neutral with regard to jurisdictional claims in published maps and institutional affiliations.

Ready to submit your research? Choose BMC and benefit from:

- fast, convenient online submission

- thorough peer review by experienced researchers in your field

- rapid publication on acceptance

- support for research data, including large and complex data types

- gold Open Access which fosters wider collaboration and increased citations

- maximum visibility for your research: over $100 \mathrm{M}$ website views per year

At $\mathrm{BMC}$, research is always in progress.

Learn more biomedcentral.com/submissions 